\title{
Multiband Optical and Near-Infrared Properties of Faint Submillimeter Galaxies with
} Serendipitous ALMA Detections

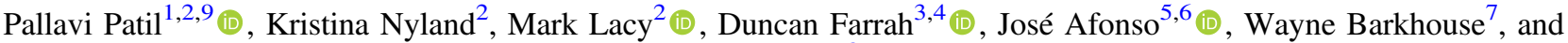 \\ Jason Surace ${ }^{8}$ (i) \\ ${ }^{1}$ Department of Astronomy, University of Virginia, 530 McCormick Road, Charlottesville, VA 22903, USA; pp3uq@ virginia.edu \\ ${ }^{2}$ National Radio Astronomy Observatory, 520 Edgemont Road, Charlottesville, VA 22903, USA \\ ${ }^{3}$ Department of Physics and Astronomy, University of Hawaii, 2505 Correa Road, Honolulu, HI 96822, USA \\ ${ }^{4}$ Institute for Astronomy, 2680 Woodlawn Drive, University of Hawaii, Honolulu, HI 96822, USA \\ ${ }^{5}$ Instituto de Astrofísica e Ciências do Espaço, Universidade de Lisboa, OAL, Tapada da Ajuda, PT1349-018 Lisboa, Portugal \\ ${ }^{6}$ Departamento de Física, Faculdade de Ciências, Universidade de Lisboa, Edifício C8, Campo Grande, PT1749-016 Lisbon, Portugal \\ ${ }^{7}$ Department of Physics and Astrophysics, University of North Dakota, Grand Forks, ND 58202-7129, USA \\ ${ }^{8}$ California Institute of Technology, 1200 E. California Boulevard, Pasadena, CA 91125, USA \\ Received 2018 June 6; revised 2018 November 18; accepted 2018 December 9; published 2019 January 25
}

\begin{abstract}
We present a catalog of 26 faint submillimeter galaxies (SMGs) in the XMM Large Scale Structure (XMM-LSS) field identified by cross-matching serendipitously detected sources in archival pre-Atacama Large Millimeter Array (ALMA) band 6 and 7 data with multiband near-infrared (NIR) and optical data from the Spitzer Extragalactic Representative Volume Survey, the VISTA Deep Extragalactic Survey, the Canada-France-Hawaii Telescope Legacy Large Survey, and the Hyper Suprime-Cam Subaru Strategic Program. Of the 26 SMGs in our sample, 15 are identified here for the first time. The majority of the sources in our sample (16/26) have faint submillimeter fluxes $\left(0.1 \mathrm{mJy}<S_{1 \mathrm{~mm}}<1 \mathrm{mJy}\right)$. In addition to the 26 SMGs with multiband optical and NIR detections, there are 60 highly reliable $(>5 \sigma)$ ALMA sources with no counterpart in any other band down to an Infrared Array Camera (IRAC) [4.5] $A B$ magnitude of $\approx 23.7$. To further characterize the 26 galaxies with both ALMA and optical/NIR counterparts, we provide 13-band forced photometry for the entire catalog using the Tractor and calculate photometric redshifts and rest-frame colors. The median redshift of our sample is $\langle z\rangle=2.66$. We find that our sample galaxies have bluer colors compared to bright SMGs, and the UVJ color plot indicates that their colors are consistent with main-sequence star-forming galaxies. Our results provide new insights into the nature of the faint population of SMGs and also highlight opportunities for galaxy evolution studies based on archival ALMA data.
\end{abstract}

Key words: galaxies: evolution - galaxies: high-redshift - galaxies: photometry - galaxies: star formation submillimeter: galaxies

Supporting material: figure sets

\section{Introduction}

Dusty star-forming galaxies (DSFGs) are a class of galaxies enshrouded in dust that have star formation rates of at least a few tens of solar masses per year (Casey et al. 2014). A characteristic feature of this class is the bright dust emission at far-infrared (IR) wavelengths, which is the reradiated optical/ultraviolet (UV) light from star-forming regions. Their discovery became possible by the advances in IR instruments during the 1980s-1990s, and the Infra-Red Astronomical Satellite allowed a large number of detections of luminous and ultraluminous infrared galaxies. Furthermore, the Cosmic Background Explorer satellite was the first to measure the cosmic infrared background (CIB) light and establish the overall importance of the DSFG population. The results from Cosmic Background Explorer showed that the energy density of the CIB emission is comparable to that of the optical and UV background light (Hauser et al. 1998; Dole et al. 2006). Studies in the late 1990s (Smail et al. 1997; Barger et al. 1998; Hughes et al. 1998; Blain et al. 2002, and references therein) identified the galaxies responsible for the CIB submillimeter/ millimeter emission. These galaxies, popularly known as submillimeter galaxies (SMGs; Blain et al. 2002), belong to the more general class of DSFGs.

\footnotetext{
${ }^{9}$ Reber Pre-Doctoral Fellow.
}

SMGs are extremely luminous galaxies $\left(L_{\mathrm{IR}}>10^{12} L_{\odot}\right)$ with star formation rates up to $1000 M_{\odot} \mathrm{yr}^{-1}$ (Ivison et al. 1998; Smail et al. 2002; Chapman et al. 2004; Barger et al. 2012; Swinbank et al. 2014; Michałowski et al. 2017; Simpson et al. 2017). They are most likely undergoing a merger (Engel et al. 2010; Alaghband-Zadeh et al. 2012; Ivison et al. 2012; Fu et al. 2013; Chen et al. 2015; Oteo et al. 2016) and have a median redshift, $\langle z\rangle \sim 2$-3 (Chapman et al. 2002, 2005; Wardlow et al. 2011; Simpson et al. 2014; Miettinen et al. 2015; Chen et al. 2016a). Although early studies of SMGs only utilized observation windows at $850 \mu \mathrm{m}$ and $1.1 \mathrm{~mm}$ because of atmospheric transmission, the negative $k$-correction helped to probe SMGs up to high redshift $(z \sim 5)$. For a given luminosity, the dimming of the flux with increasing redshift is balanced by the shifting of the peak of the spectral energy distribution (SED) into the observing window (Franceschini et al. 1991; Blain et al. 2002; See Figure 4 in Blain et al. 2002). Therefore, the flux remains approximately constant for redshifts up to $z \sim 8$.

Single-dish instruments, such as the Submillimetre Common User Bolometer Array (SCUBA; e.g., Smail et al. 1997; Barger et al. 1998; Hughes et al. 1998) on the James Clark Maxwell Telescope (JCMT), AzTEC (Ezawa et al. 2004; Perera et al. 2008; Austermann et al. 2010; Scott et al. 2010, 2012; Aretxaga et al. 2011) on the Atacama Submillimeter Telescope Experiment, the Large Apex Bolometer Camera (LABOCA) on the Atacama 
Pathfinder Experiment (Siringo et al. 2009; Weiß et al. 2009), Bolocam on the Caltech Submillimeter Observatory (Laurent et al. 2005), the Max Planck Millimeter Bolometer (MAMBO; Greve et al. 2004; Bertoldi et al. 2007) on the Institut de radioastronomie millimétrique $30 \mathrm{~m}$ telescope, and SCUBA-2 on JCMT (Casey et al. 2013; Chen et al. 2013a; Geach et al. 2013, 2017), identified a significant number of SMGs. However, large beam sizes $\left(15^{\prime \prime}\right.$ for SCUBA at $850 \mu \mathrm{m}$ and $11^{\prime \prime}$ for MAMBO-1 at $1.2 \mathrm{~mm}$ ) prevented accurate multiwavelength counterpart identification. A few indirect techniques were used to identify multiwavelength counterparts, such as using the radio-far-IR correlation to find targets in radio interferometric observations (Ivison et al. 2007), $24 \mu \mathrm{m}$ Multiband Imaging Photometer for Spitzer observations from Spitzer (e.g., Pope et al. 2006), and assigning probabilities to the possible optical/near-IR (NIR) counterparts (e.g., Ivison et al. 2002; Dunlop et al. 2004; Biggs et al. 2011; Chapin et al. 2011; Smith et al. 2011; Kim et al. 2012; Alberts et al. 2013). These techniques have limitations because not all counterparts can be accurately identified owing to large beam sizes of single-dish telescopes. Therefore, interferometric observation is required to obtain a bias-free sample of submillimeter sources with subarcsecond positional accuracy.

Number count studies revealed that a significant population was missed by the single dish, as well as space-based observations at submillimeter and millimeter wavelengths (Lagache et al. 2005). On the other hand, most of the population contributing to the CIB at wavelengths less than $200 \mu \mathrm{m}$ was already identified and found to reside at $z \sim 1$ (Viero et al. 2013). Number counts and models showed that the redshift of the dominant contributor to the CIB increases with increasing wavelength (Lagache et al. 2005). This indicates the need for high sensitivity and finer spatial resolution surveys. During the pre-Atacama Large Millimeter Array (ALMA) era, $1.1 \mathrm{~mm}$ surveys were mainly conducted with AzTEC (Perera et al. 2008; Austermann et al. 2010; Scott et al. 2010, 2012; Aretxaga et al. 2011; Hatsukade et al. 2011) and about $10 \%-20 \%$ of the CIB was resolved (Scott et al. 2010; Hatsukade et al. 2011). In contrast, $850 / 870 \mu \mathrm{m}$ surveys using SCUBA, the LABOCA has resolved up to $50 \%$ of the CIB (Blain et al. 1999; Coppin et al. 2006; Weiß et al. 2009). ${ }^{10}$ The high confusion limit limited the detection threshold to $\sim 1-2 \mathrm{mJy}$ at $850 \mu \mathrm{m}$. Studies of gravitationally lensed SMGs allowed probing of the faint population (Smail et al. 1997; Cowie et al. 2002; Knudsen et al. 2008; Johansson et al. 2011; Chen et al. 2013a, 2013b, 2014; Hsu et al. 2016). However, these studies were limited by the small number of statistics and by uncertainties in the lensing models for the clusters. In summary, all of the previous results have shown that the major contributors of the CIB at $850 \mu \mathrm{m}$ and $1.1 \mathrm{~mm}$ have flux densities fainter than $1 \mathrm{mJy}$. Such galaxies would correspond to normal galaxies, or luminous infrared galaxies (with luminosities $L<10^{12} L_{\odot}$ ).

It is now possible to study this fainter population, the so-called faint SMGs, because ALMA provides subarcsecond resolution and high sensitivity at submillimeter and millimeter wavelengths. However, the small field of view of ALMA makes large surveys challenging. Several groups have tried different approaches to optimize ALMA's resources and search for the faint population. One approach is to look for serendipitous detections using archival observations obtained for other scientific goals (Hatsukade et al. 2013; Ono et al. 2014; Carniani et al. 2015; Fujimoto et al. 2016; Oteo et al. 2016). Another

\footnotetext{
${ }^{10}$ We have provided only a selected number of references here. Refer to Section 3 in Casey et al. (2014) for a complete list.
}

approach is to observe a contiguous field using ALMA (Kohno et al. 2016; Dunlop et al. 2017; González-López et al. 2017; Franco et al. 2018).

A third approach made use of the available deep optical-NIR surveys to develop a triple-color selection technique for identification faint galaxies (Chen et al. 2016a). These pilot studies are attempting to address the contribution of faint SMGs to the CIB, their multiwavelength counterparts, and their role in shaping galaxy formation. The number count studies revealed that faint SMGs contribute significantly to the extragalactic background light at $1.1 \mathrm{~mm}$. Fujimoto et al. (2016) found that the contribution of faint SMGs $(0.02 \mathrm{mJy}<$ $S_{1.2 \mathrm{~mm}}<1 \mathrm{mJy}$ ) can account for all of the CIB at $1.2 \mathrm{~mm}$. If we assume a median redshift, $z \sim 2$, the IR luminosities of faint SMGs are expected to be in the range $10^{11-12} L_{\odot}$ (Chen et al. 2016a). Therefore, this population bridges the gap between extreme star-forming galaxies (bright SMGs) and optical-color selected galaxies with moderate star formation rates, such as Lyman Break galaxies ${ }^{11}$ and star-forming BzK galaxies $^{12}$ (Chen et al. 2016a).

On the basis of optical-NIR color-color plots, the faint SMGs from Fujimoto et al. (2016) were found to represent Lyman Break galaxies/star-forming BzK galaxies. However, these studies suffer from small number statistics, and very little is known about this newly discovered population. Robust estimates of demographics, such as number counts, the redshift distribution, the contribution to the cosmic star formation rate density, the nature of their multiwavelength counterparts, and the star formation rate distribution are some of the key issues that need to be addressed.

In this article, we study faint SMGs in the XMM Large-Scale Structure (XMM-LSS) field with multiband optical and IR survey data that have serendipitous submillimeter counterparts identified in archival ALMA observations. Many of the sources identified in our sample are faint SMGs, with $\sim 1 \mathrm{~mm}$ fluxes below $1 \mathrm{mJy}$. We investigate the properties of this cosmologically important galaxy population by performing multiband forced photometry to obtain photometric redshifts and place constraints on star formation. Our study also highlights the growing opportunities for probing high-redshift galaxy properties and gaining new insights on cosmic assembly by mining the ALMA archive.

In Section 2, we describe our sample selection procedure. Details on our reduction of the archival ALMA data, sourcefinding strategy, multiband photometric catalog construction, and photometric redshift determination are given in Section 3. We discuss the multiwavelength source properties of our sample in Section 4 and provide a summary of our results in Section 5. Throughout this study, we adopt a $\Lambda$ CDM cosmology with $\Omega_{\mathrm{M}}=0.3, \Omega_{\Lambda}=0.7$, and $H_{0}=70 \mathrm{~km} \mathrm{~s}^{-1} \mathrm{Mpc}^{-1}$.

\section{Sample}

\subsection{Optical and Infrared Data}

Our sample is drawn from the XMM-LSS field. The XMM-LSS field includes abundant multiband data at optical and IR wavelengths from a variety of wide-field surveys. Of particular importance is the availability of deep Spitzer IRAC observations at

\footnotetext{
${ }^{11}$ See Steidel et al. (1996) for more on the properties of Lyman Break galaxies.

${ }^{12}$ See Daddi et al. (2004) for the formal definition of star-forming BzK galaxies.
} 


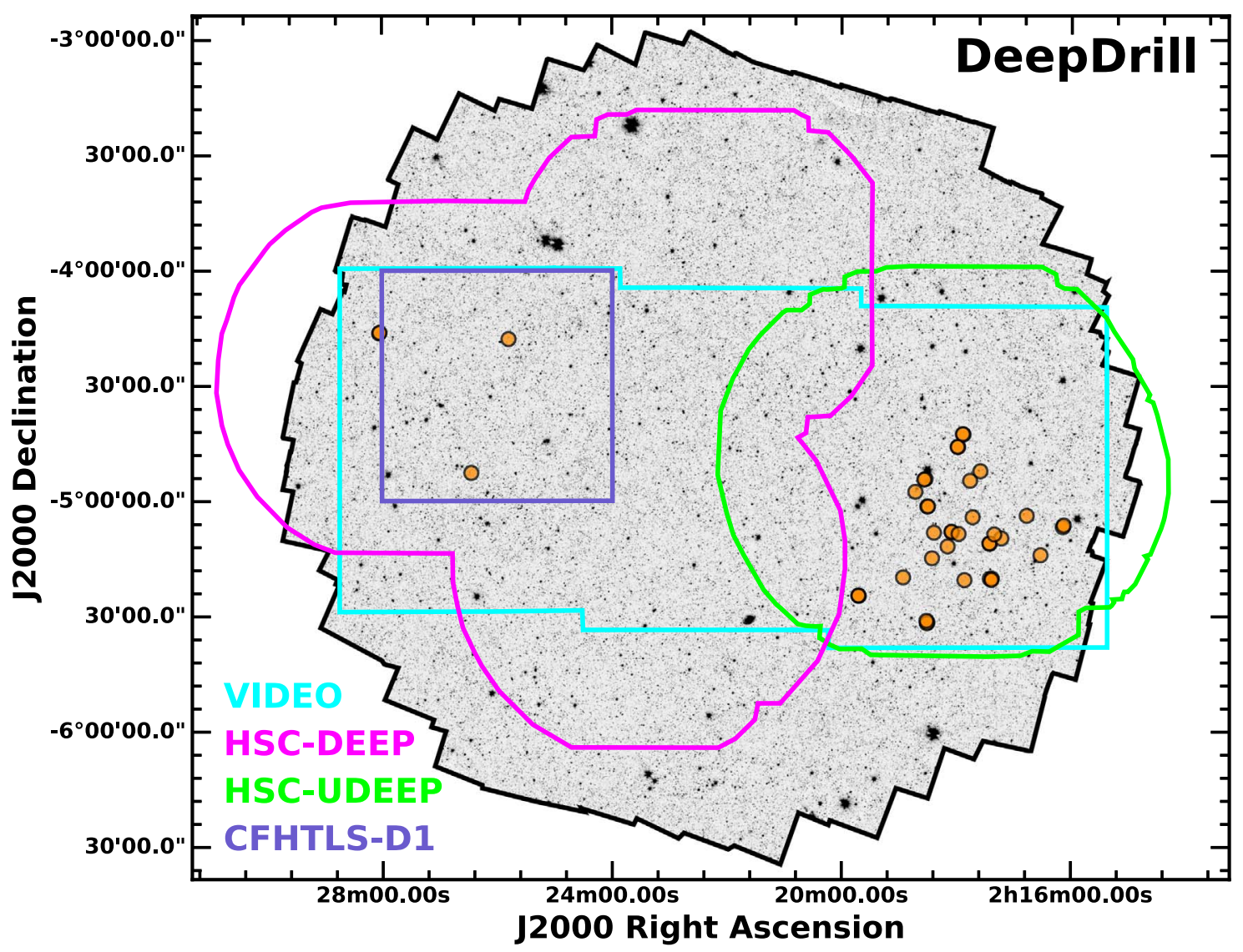

Figure 1. The multiband coverage by the surveys used in our study in XMM-LSS field. The grayscale image is the DeepDrill $3.6 \mu \mathrm{m}$ mosaic, the cyan region traces the VIDEO coverage, the green region shows the HSC Ultra Deep tier, the magenta region denotes the HSC deep tier, and the purple region shows the CFHTLS coverage. The orange circles indicate the ALMA sources with optical/NIR counterparts identified in this study.

3.6 and $4.5 \mu \mathrm{m}$ from the Spitzer Extragalactic Representative Volume Survey (SERVS; Mauduit et al. 2012) and DeepDrill (P.I. Mark Lacy). SERVS is a postcryogenic IRAC survey of five well-studied astronomical deep fields with a depth of $2 \mu \mathrm{Jy}$ and a total sky footprint of $18 \mathrm{deg}^{2}$. The DeepDrill survey expands upon the sky coverage of SERVS and provides deep IRAC imaging in three of the four predefined Deep Drilling Fields for the Large Synoptic Survey Telescope over an area of $38.4 \mathrm{deg}^{2}\left(\sim 1 \mathrm{Gpc}^{3}\right.$ at $z>2$ ).

Observations at 3.6 and $4.5 \mu \mathrm{m}$ are crucial for detecting restframe optical emission from galaxies at $z>4$, and, when combined with additional photometry at optical and NIR wavelengths, provide constraints on important galaxy properties, such as photometric redshift. The SERVS and DeepDrill observations in the XMM-LSS field are complemented by additional NIR data from the ground-based VISTA Deep Extragalactic Survey (VIDEO; Jarvis et al. 2013) in the $Z, Y, J$, $H$, and $K s$ bands. The optical, wide-field data are available from the Canada-France-Hawaii Telescope Legacy Survey Wide field 1 (CFHTLS-W1; Gwyn 2012) and multiple tiers from the first data release of the Hyper Suprime-Cam Subaru Strategic Program (HSC; Aihara et al. 2018). We include the NIR data from SERVS/DeepDrill and VIDEO, as well as broadband optical data from HSC in the grizy filter set and data from CFHTLS-W1 in the $u$-band in our analysis. Thus, we have a total of 13 bands available for determining photometric redshifts. We illustrate the sky coverage of these surveys in Figure 1.

\subsection{Archival ALMA Data}

We mined the ALMA archive to search for continuum observations within the XMM-LSS field that were publicly available as of 2017 July. We required the following criteria for selecting the archival ALMA data: 1) observations performed in band $6(211-275 \mathrm{GHz})$ or band $7(275-370 \mathrm{GHz}), 2)$ a source integration time longer than $150 \mathrm{~s}$ to ensure sufficient sensitivity to the inherently faint SMG population, and 3) an angular resolution of $\theta_{\text {FWHM }}>0$ ". 4 to ensure adequate surface brightness sensitivity. After evaluating the central depths for all the available programs making the resolution cut, we found that our integration time criterion leads to a maximum $1 \sigma$ rms noise of $150 \mu \mathrm{Jy}$ beam $^{-1}$ in band 6 and $300 \mu \mathrm{Jy}$ beam ${ }^{-1}$ in band 7 . Given the nature of our source search, the source depth is variable $^{13}$ and, therefore, finding a complete census of faint SMG is not a primary goal of our search in this article. We aim to find as many faint galaxies as we can to build up a large sample of the faint population having comprehensive multiwavelength coverage. Such a sample will allow us to conduct detailed studies that will help guide future statistical studies.

\footnotetext{
${ }^{13}$ The source search depth depends on the integration time, as well as the distance of the source from the ALMA pointing center. As the noise increases toward the edge of the field of view because of the primary-beam response, the sensitivity is variable within each pointing. Therefore, specifying the completeness limit would not be meaningful for our study, which aims to explore the properties of the faint SMG population rather perform a statistically complete analysis.
} 
Our search identified 75 continuum maps from nine different projects, ${ }^{14}$ all but one observed in band 6 . The typical rms noise levels for band $6(\sim 1.2 \mathrm{~mm})$ and band $7(870 \mu \mathrm{m})$ are $15-140 \mu \mathrm{Jy}_{\text {beam }}{ }^{-1}$ and $200-300 \mu \mathrm{Jy} \mathrm{beam}^{-1}$, respectively. Therefore, we note that the image depth is not uniform throughout our sample. Table 4 summarizes the list of ALMA pointings considered for our study. The angular resolutions of the archival data ranged from 0 ". 53 to 1 ". 46 .

\section{Data Analysis}

\subsection{ALMA}

\subsubsection{Calibration and Imaging}

We reduced the archival ALMA data using the Common Astronomy Software Applications (McMullin et al. 2007) package. We ran the calibration scripts that are provided along with the raw data from the ALMA archive. The calibration scripts include a priori flagging, bandpass calibration, flux calibration, and gain calibration. The calibrated products were examined for further flagging in the UV-plane as well as the image plane. We found that the provided script had flagged most of the bad data and very little additional flagging was required.

We used the CLEAN task to form and deconvolve images with the recommended parameters provided by the ALMA observatory. Specifically, CLEAN was run in multifrequency synthesis mode with nterms $=1$. The weighting was either natural or Briggs weighting with a robust parameter of 0.5, and was determined on a case-by-case basis. Maps with bright targets were self-calibrated (using one round of phase-only selfcalibration) and reimaged.

\subsubsection{Source Extraction}

The Python Blob Detector and Source Finder (PyBDSF) tool was used to extract sources from the ALMA maps (Mohan \& Rafferty 2015). Continuum maps without primary-beam corrections were used to search for sources. The algorithm looks for image pixels above a specified threshold (here we used threshpix $=3.0$ ). Contiguous pixels above the threshold with a minimum size of one-third of the synthesized beam are formed into a single island. An island is considered a valid source if a single- or multiple-component Gaussian fit is successful.

We have found that PyBDSF works well with the default parameters. However, we set the pixel threshold parameter to $3.5 \sigma$ instead of $5 \sigma$, because the default threshold was too conservative to probe the fainter population. By lowering the threshold, we are increasing the contamination, but prior source position information from the multiwavelength optical/IR data will eliminate most of the spurious sources. The preliminary catalog contains all extracted sources, including the science targets of the proposed observations. We have also removed the sources lying at a distance larger than the radius where the primary-beam sensitivity drops to $10 \%$ of its maximum, and four strongly lensed galaxies that were the targets of some of the original observations. Although PyBDSF provides estimates of several parameters, including total and peak flux densities, convolved and deconvolved source sizes, and

\footnotetext{
14 The nine public archival ALMA projects were undertaken for entirely different science goals. Brief descriptions of those goals are given in Appendix A.
}

uncertainties on each parameter, we chose to use it only to identify ALMA source position. Those source positions were then cross-matched with the multiband optical/IR data (see Section 3.2).

To avoid known issues with flux overestimation of faint sources with PyBDSF (Hopkins et al. 2015), we used the JMFIT task from the Astronomical Image Processing Software to measure source fluxes and their uncertainties. For each ALMA source, we used JMFIT to fit a two-dimensional, single-component Gaussian at the position from our source extraction with PyBDSF. All JMFIT measurements were based on the primary-beam-corrected ALMA images. We have tabulated the ALMA fluxes of our sample sources in Table 1.

\subsubsection{Detection Threshold}

Some spurious detections are likely to contaminate the source catalog, assuming pure Gaussian-like noise. Therefore, it is necessary to determine the signal-to-noise ratio [Q: Please note that the journal prefers to use " $\mathrm{S} / \mathrm{N}$ " as the abbreviation for "signal-to-noise ratio" because "SNR" is reserved for "supernova remnant."'](S/N) cutoff at which an optimal compromise is made between minimizing the number of spurious sources and maintaining a reasonable level of completeness for faint objects. One way to quantify the level of spurious source contamination is to perform a negative peak analysis (Hatsukade et al. 2013; Ono et al. 2014; Carniani et al. 2015; Fujimoto et al. 2016). To accomplish this, we multiplied each ALMA image by -1 and ran PyBDSF using the same input parameters as the ones used in the original source extraction. We then plotted separate $\mathrm{S} / \mathrm{N}$ distributions for all sources extracted from the negative and positive peak analyses.

If a given map only contains noise and no real emission, then the total number of sources in the positive and negative maps would be approximately the same. This will result in a similar source distribution as a function of $\mathrm{S} / \mathrm{N}$. However, when real sources are present, we will start to see an excess of positive sources over negative sources above a certain $\mathrm{S} / \mathrm{N}$ value. The detection threshold for the source catalog can then be chosen at a certain value, after which the number of positive detections is greater than the number of negative ones. Figure 2 shows the number of sources extracted from both the positive and negative maps from our work. On the basis of this figure, we selected a detection threshold of $3.9 \sigma$ for the archival ALMA data. Once this threshold was applied, our ALMA catalog was reduced to 176 objects. We then cross-matched this catalog with the optical-NIR photometry (see Section 3.2) and found 26 faint SMGs with counterparts within a search radius of $1^{\prime \prime}$.

To check the fidelity of our source selection, we performed the same counterpart matching steps for our negative ALMA source catalog and found seven of 88 sources with optical-NIR counterparts, whereas we found optical-NIR cross-matches for 26 of 176 sources in the positive source search. Thus, the combination of our detection threshold in the ALMA data and an optical-NIR counterpart leads to a significantly greater level of fidelity compared to the level (50\%) that the 3.9 $\sigma$ ALMA detection threshold alone would provide.

In extracting sources from the negative maps, we did find a few targets with multi-Gaussian structures. Because we do not expect to see any complex sources, this could be due to image artifacts. Therefore, we excluded sources with such structures from our catalog. We note that we have not estimated the formal completeness of our ALMA catalog, nor are we 
Table 1

ALMA Source Catalog

\begin{tabular}{|c|c|c|c|c|c|c|c|c|c|c|}
\hline (1) & $\begin{array}{l}\text { IAU ID } \\
\text { (2) }\end{array}$ & $\begin{array}{l}\text { Field } \\
\text { (3) }\end{array}$ & $\begin{array}{l}\text { R.A. } \\
\text { (J2000) } \\
\text { (4) }\end{array}$ & $\begin{array}{l}\text { Decl. } \\
(\mathrm{J} 2000) \\
(5)\end{array}$ & $\begin{array}{c}\lambda_{\text {obs }} \\
(\mathrm{mm}) \\
(6)\end{array}$ & $\begin{array}{l}S_{\text {入obs }} \\
(\mathrm{mJy}) \\
(7)\end{array}$ & $\begin{array}{l}z_{\text {phot }} \\
(8)\end{array}$ & $\begin{array}{c}z_{\text {archival }} \\
\text { (9) }\end{array}$ & $\begin{array}{c}\text { References } \\
\text { (10) }\end{array}$ & $\begin{array}{c}\text { ALMA References } \\
\text { (11) }\end{array}$ \\
\hline 191313 & $\mathrm{~J} 0226-0452$ & WMH5 & $02^{\mathrm{h}} 26^{\mathrm{m}} 27^{\mathrm{s}} .00$ & $-04^{\mathrm{d}} 52^{\mathrm{m}} 38^{\mathrm{s}} .38$ & 1.14 & $0.18 \pm 0.04$ & $4.87_{-0.26}^{+1.07}$ & $6.068^{\mathrm{a}}$ & 4 & $\mathrm{a}$ \\
\hline 309436 & J0225-0417 & XMMF6 & $02^{\mathrm{h}} 25^{\mathrm{m}} 48^{\mathrm{s}} .03$ & $-04^{\mathrm{d}} 17^{\mathrm{m}} 48^{\mathrm{s}} .96$ & 0.87 & $1.33 \pm 0.53$ & $2.77_{-0.25}^{+0.14}$ & $\ldots$ & $\ldots$ & $\ldots$ \\
\hline 315617 & J0228-0416 & CLM1 & $02^{\mathrm{h}} 28^{\mathrm{m}} 02^{\mathrm{s}} .58$ & $-04^{\mathrm{d}} 16^{\mathrm{m}} 06^{\mathrm{s}} .99$ & 1.16 & $0.17 \pm 0.03$ & $0.29_{-0.09}^{+0.26}$ & $\ldots$ & $\ldots$ & $\ldots$ \\
\hline 477360 & J0219-0524.63 & XMMF16 & $02^{\mathrm{h}} 19^{\mathrm{m}} 42^{\mathrm{s}} .63$ & $-05^{\mathrm{d}} 24^{\mathrm{m}} 37^{\mathrm{s}} .07$ & 0.87 & $9.66 \pm 1.22$ & $1.75_{-0.11}^{+0.16}$ & 2.048 & 1 & $\mathrm{~b}$ \\
\hline 814024 & J0219-0524.52 & XMMF16 & $02^{\mathrm{h}} 19^{\mathrm{m}} 42^{\mathrm{s}} \cdot 52$ & $-05^{\mathrm{d}} 24^{\mathrm{m}} 41^{\mathrm{s}} .27$ & 0.87 & $2.61 \pm 0.50$ & $2.49_{-0.42}^{+0.46}$ & $\ldots$ & $\ldots$ & $\cdots$ \\
\hline 842544 & J0219-0524.77 & XMMF16 & $02^{\mathrm{h}} 19^{\mathrm{m}} 42^{\mathrm{s}} .77$ & $-05^{\mathrm{d}} 24^{\mathrm{m}} 36^{\mathrm{s}} .43$ & 0.87 & $2.81 \pm 0.50$ & $2.57_{-0.24}^{+0.42}$ & 1.489 & 1 & $\mathrm{~b}$ \\
\hline 911805 & J0219-0524.84 & XMMF16 & $02^{\mathrm{h}} 19^{\mathrm{m}} 42^{\mathrm{s}} .84$ & $-05^{\mathrm{d}} 24^{\mathrm{m}} 35^{\mathrm{s}} .11$ & 0.87 & $2.69 \pm 0.49$ & $2.97_{-0.12}^{+0.12}$ & 1.489 & 1 & $\mathrm{~b}$ \\
\hline 932297 & J0217-0520 & SXDF1100.277 & $02^{\mathrm{h}} 17^{\mathrm{m}} 23^{\mathrm{s}} .50$ & $-05^{\mathrm{d}} 20^{\mathrm{m}} 08^{\mathrm{s}} .62$ & 1.13 & $2.28 \pm 0.41$ & $3.62_{-0.12}^{+0.12}$ & $\ldots$ & $\ldots$ & $\ldots$ \\
\hline 935442 & J0218-0519 & SXDF.220GHZ & $02^{\mathrm{h}} 18^{\mathrm{m}} 56^{\mathrm{s}} .10$ & $-05^{\mathrm{d}} 19^{\mathrm{m}} 51^{\mathrm{s}} .00$ & 1.33 & $0.10 \pm 0.03$ & $1.38_{-0.12}^{+0.09}$ & $1.48_{-0.07}^{+0.10}$ & 1,2 & $\ldots$ \\
\hline 971686 & J0217-0511 & UDS16 & $02^{\mathrm{h}} 17^{\mathrm{m}} 25^{\mathrm{s}} \cdot 72$ & $-05^{\mathrm{d}} 11^{\mathrm{m}} 03^{\mathrm{s}} \cdot 17$ & 1.24 & $0.10 \pm 0.03$ & $2.73_{-0.23}^{+0.20}$ & $0.27_{-0.05}^{+2.66}$ & 2 & $\ldots$ \\
\hline 978351 & J0217-0510 & UDS16 & $02^{\mathrm{h}} 17^{\mathrm{m}} 26^{\mathrm{s}} .10$ & $-05^{\mathrm{d}} 10^{\mathrm{m}} 58^{\mathrm{s}} \cdot 20$ & 1.24 & $0.95 \pm 0.12$ & $2.12_{-0.34}^{+0.14}$ & $1.55_{-0.07}^{+0.20}$ & 1,2 & $\ldots$ \\
\hline 987090 & J0217-0508 & SXDF1100.027 & $02^{\mathrm{h}} 17^{\mathrm{m}} 20^{\mathrm{s}} .95$ & $-05^{\mathrm{d}} 08^{\mathrm{m}} 37^{\mathrm{s}} .17$ & 1.13 & $1.39 \pm 0.18$ & $2.95_{-0.60}^{+0.10}$ & $2.80_{-0.70}^{+0.48}$ & 1,5 & $\mathrm{c}$ \\
\hline 993676 & J0216-0506 & SXDS5.28019 & $02^{\mathrm{h}} 16^{\mathrm{m}} 08^{\mathrm{s}} \cdot 51$ & $-05^{\mathrm{d}} 06^{\mathrm{m}} 15^{\mathrm{s}} .89$ & 1.27 & $0.18 \pm 0.10$ & $1.29_{-0.08}^{+0.05}$ & $1.348^{\mathrm{a}}$ & $1,2,6$ & $\mathrm{~d}, \mathrm{e}$ \\
\hline 998253 & J0217-0508 & HIMIKO & $02^{\mathrm{h}} 17^{\mathrm{m}} 58^{\mathrm{s}} .28$ & $-05^{\mathrm{d}} 08^{\mathrm{m}} 30^{\mathrm{s}} .64$ & 1.16 & $0.59 \pm 0.04$ & $1.14_{-0.08}^{+0.11}$ & $1.09_{-0.04}^{+0.03}$ & 1,2 & $\mathrm{f}$ \\
\hline 1002990 & J0216-0503 & SXDF1100.013 & $02^{\mathrm{h}} 16^{\mathrm{m}} 47^{\mathrm{s}} \cdot 10$ & $-05^{\mathrm{d}} 03^{\mathrm{m}} 44^{\mathrm{s}} \cdot 54$ & 1.13 & $1.46 \pm 0.18$ & $3.03_{-2.27}^{+0.23}$ & $\ldots$ & $\ldots$ & $\cdots$ \\
\hline 1017745 & J0218-0501 & SXDF1100.039 & $02^{\mathrm{h}} 18^{\mathrm{m}} 30^{\mathrm{s}} .98$ & $-05^{\mathrm{d}} 01^{\mathrm{m}} 23^{\mathrm{s}} \cdot 22$ & 1.13 & $0.49 \pm 0.17$ & $0.42_{-0.11}^{+0.10}$ & $0.49_{-0.03}^{+0.03}$ & 1,2 & $\ldots$ \\
\hline 1048801 & J0217-0454 & SXDS1.59863 & $02^{\mathrm{h}} 17^{\mathrm{m}} 46^{\mathrm{s}} \cdot 28$ & $-04^{\mathrm{d}} 54^{\mathrm{m}} 39^{\mathrm{s}} .77$ & 1.23 & $0.69 \pm 0.20$ & $2.29_{-1.10}^{+0.10}$ & $1.456^{\mathrm{a}}$ & $1,2,3$ & $\mathrm{~d}, \mathrm{e}$ \\
\hline 1094072 & J0217-0445 & SXDF1100.230 & $02^{\mathrm{h}} 17^{\mathrm{m}} 59^{\mathrm{s}} \cdot 38$ & $-04^{\mathrm{d}} 45^{\mathrm{m}} 53^{\mathrm{s}} .13$ & 1.13 & $1.54 \pm 0.14$ & $3.92_{-0.27}^{+0.20}$ & $3.50_{-0.18}^{+0.40}$ & $1,2,5$ & $\mathrm{c}$ \\
\hline 1106738 & J0217-0442 & SXDS2.22198 & $02^{\mathrm{h}} 17^{\mathrm{m}} 53^{\mathrm{s}} .17$ & $-04^{\mathrm{d}} 42^{\mathrm{m}} 39^{\mathrm{s}} .61$ & 1.27 & $0.44 \pm 0.10$ & $0.03_{-0.02}^{+0.04}$ & $\ldots$ & $\ldots$ & $\mathrm{d}$ \\
\hline 1266981 & J0217-0520 & SXDF1100.277 & $02^{\mathrm{h}} 17^{\mathrm{m}} 23^{\mathrm{s}} .95$ & $-05^{\mathrm{d}} 20^{\mathrm{m}} 28^{\mathrm{s}} .02$ & 1.13 & $0.55 \pm 0.14$ & $2.66_{-0.10}^{+0.11}$ & $2.54_{-0.40}^{+0.43}$ & 1 & $\cdots$ \\
\hline 1302443 & J0218-0501 & SXDF1100.039 & $02^{\mathrm{h}} 18^{\mathrm{m}} 30^{\mathrm{s}} .23$ & $-05^{\mathrm{d}} 01^{\mathrm{m}} 21^{\mathrm{s}} .19$ & 1.13 & $0.84 \pm 0.17$ & $3.21_{-0.41}^{+0.39}$ & $2.66_{-0.73}^{+0.94}$ & 1 & $\cdots$ \\
\hline 1302615 & J0217-0520 & SXDF1100.250 & $02^{\mathrm{h}} 17^{\mathrm{m}} 52^{\mathrm{s}} .00$ & $-05^{\mathrm{d}} 20^{\mathrm{m}} 32^{\mathrm{s}} \cdot 34$ & 1.13 & $0.97 \pm 0.14$ & $3.65_{-1.20}^{+0.81}$ & $2.79_{-1.45}^{+0.45}$ & 1 & $\ldots$ \\
\hline 1303410 & J0218-0508 & SXDF1100.109 & $02^{\mathrm{h}} 18^{\mathrm{m}} 23^{\mathrm{s}} .99$ & $-05^{\mathrm{d}} 08^{\mathrm{m}} 11^{\mathrm{s}} \cdot 40$ & 1.13 & $0.79 \pm 0.31$ & $3.16_{-0.21}^{+0.20}$ & $1.91_{-0.39}^{+0.61}$ & 1 & $\cdots$ \\
\hline 1304155 & J0217-0452 & SXDF1100.063 & $02^{\mathrm{h}} 17^{\mathrm{m}} 35^{\mathrm{s}} .50$ & $-04^{\mathrm{d}} 52^{\mathrm{m}} 11^{\mathrm{s}} .79$ & 1.13 & $0.62 \pm 0.17$ & $2.59_{-0.45}^{+0.41}$ & $\ldots$ & $\ldots$ & $\ldots$ \\
\hline 1307256 & J0218-0457 & SXDF1100.179 & $02^{\mathrm{h}} 18^{\mathrm{m}} 43^{\mathrm{s}} \cdot 41$ & $-04^{\mathrm{d}} 57^{\mathrm{m}} 33^{\mathrm{s}} .05$ & 1.13 & $0.79 \pm 0.14$ & $5.40_{-5.38}^{+0.56}$ & $\ldots$ & $\ldots$ & $\cdots$ \\
\hline 1312163 & J0217-0504 & SXDF1100.110 & $02^{\mathrm{h}} 17^{\mathrm{m}} 43^{\mathrm{s}} .58$ & $-05^{\mathrm{d}} 04^{\mathrm{m}} 10^{\mathrm{s}} \cdot 31$ & 1.13 & $2.61 \pm 0.30$ & $4.47_{-1.13}^{+1.44}$ & $4.98_{-3.14}^{+0.72}$ & 5 & $\mathrm{c}$ \\
\hline
\end{tabular}

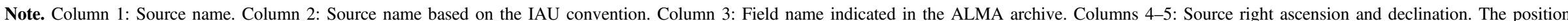

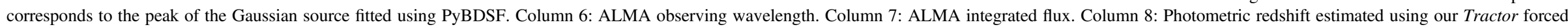

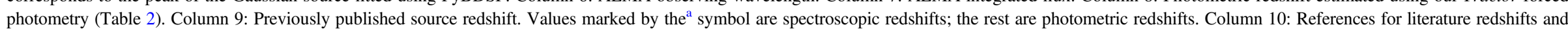

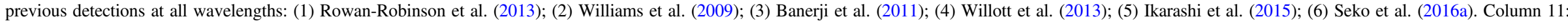

References for previous ALMA detections: (a) Willott et al. (2015); (b) Bussmann et al. (2015); (c) Ikarashi et al. (2015); (d) Fujimoto et al. (2016); (e) Hatsukade et al. (2013); (f) Ono et al. (2014).

${ }^{\mathrm{a}}$ Spectroscopic redshifts. 

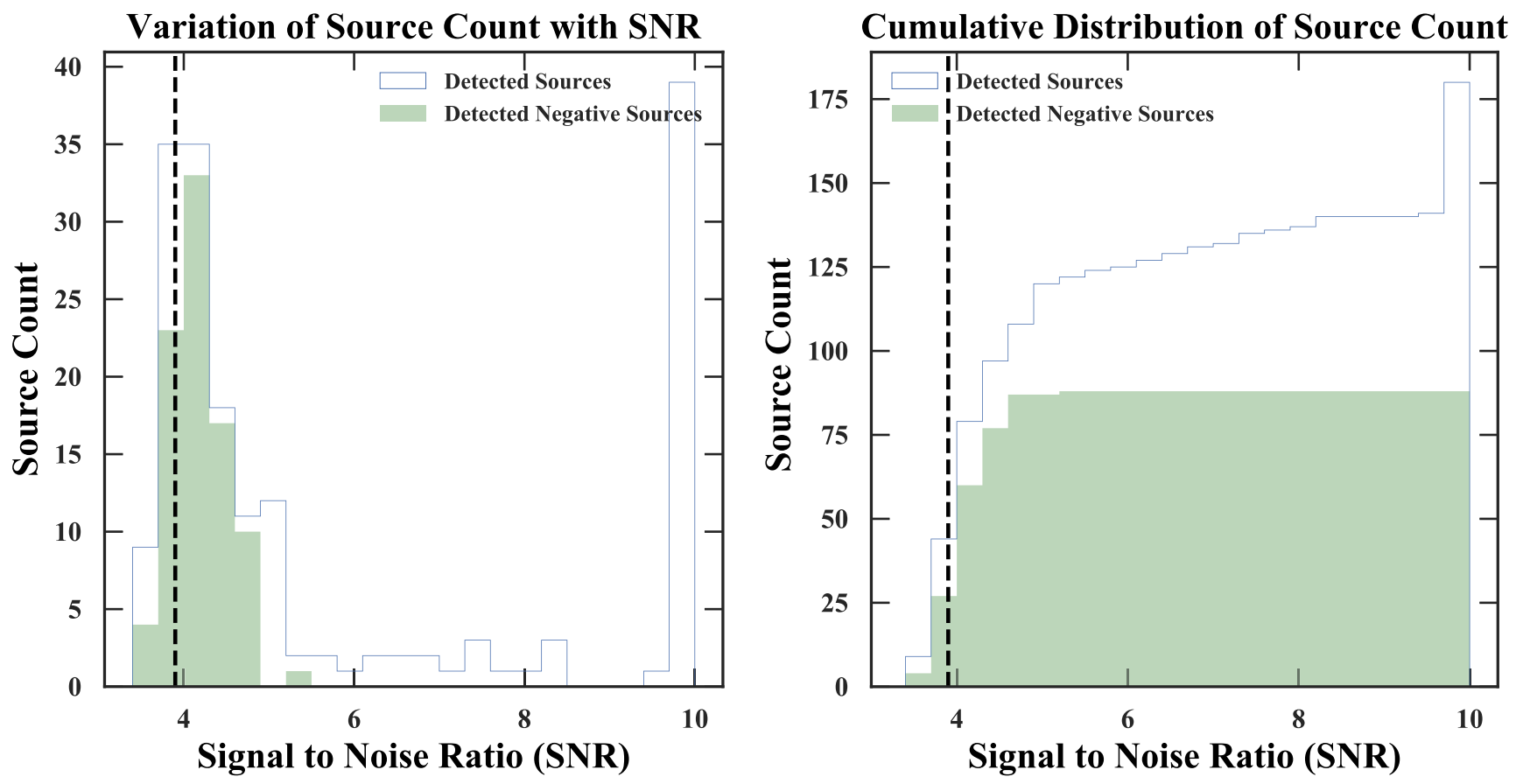

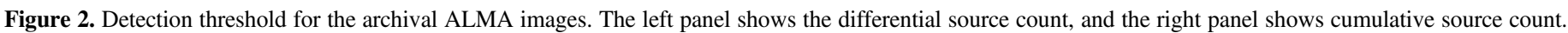
This analysis was used to select a detection threshold of $3.9 \sigma$ for the archival ALMA images.

conducting a number count analysis. Because we are limited by the availability of data in the archive, our sample will naturally be incomplete. In this article, we focus on investigating the multiwavelength properties of our sample of faint SMGs.

\subsubsection{Comparison with Previous Studies}

A few of the ALMA pointings in our sample and that of Fujimoto et al. (2016) overlap, which provides us with an opportunity to compare methodologies, source counts, and source fluxes in the respective samples. In total, 20 ALMA pointings from three different archival programs (ALMA 2011.0.00115.S, 2011.0.00648.S, and 2012.1.00934.S) are common with Fujimoto et al. (2016). The source extraction parameters for our study are different from those of Fujimoto et al. (2016). The authors have selected sources above an $\mathrm{S} / \mathrm{N}$ of $3.4 \sigma$ within a search radius having primary-beam sensitivity of $50 \%$ or larger, whereas we used $3.9 \sigma$ as an $\mathrm{S} / \mathrm{N}$ threshold and a search radius having primary-beam sensitivity greater than $10 \%$ of its maximum. In our source extraction, we found 26 sources above $3.9 \sigma$ within those 20 pointings. Fujimoto et al. (2016) found 14 sources above $3.4 \sigma$, of which six sources are above $3.9 \sigma$. Those six sources are also detected in our sample. Therefore, source counts of both the studies are consistent when same source extraction parameters are selected. Furthermore, the estimated flux densities also agree within the quoted uncertainties for those six sources.

\subsection{Multiband Forced Photometry}

To proceed with our photometric redshift and SED analysis, construction of a robust multiband photometric source catalog is necessary. However, the difference in angular resolution between the Spitzer IRAC $\left(\sim 2^{\prime \prime}\right)$ and ground-based optical/NIR survey data $\left(\lesssim 1^{\prime \prime}\right)$, coupled with the crowded nature of these observations, make the IRAC data prone to issues with source blending.
This is problematic for accurate source cross-identification between bands and reliable multiband photometry.

Recently, Nyland et al. (2017) demonstrated a means of mitigating many of the issues inherent to mixed-resolution optical/NIR data sets using a "forced photometry" approach based on the Tractor imaging modeling code (Lang et al. 2016). This code uses prior information on source position and surface brightness profile from a high-resolution, "fiducial" band, along with image calibration parameters including the point-spread function, to model the source flux in lowerresolution bands. After applying the Tractor to a one square degree test region of the XMM-LSS field, Nyland et al. (2017) found a number of improvements in the resulting multiband forced photometry compared to traditional position-matched source catalogs. In particular, they found that the Tractor forced photometry decreased susceptibility to blending issues, led to more reliable source cross-matching between bands, identified a larger number of candidate high-redshift $(z>5)$ objects, and produced more-accurate photometric redshifts when compared to available spectroscopic redshift data.

We have adopted a strategy similar to that described in Nyland et al. (2017) in constructing the optical/NIR source catalog used for determining photometric redshifts of the ALMA sources in our study. This strategy requires an initial, position-matched input catalog that is constructed by crossmatching the positions of the ALMA sources with positions from VIDEO ${ }^{15}$ using a search radius of $1^{\prime \prime}$. Thus, each source in this "VIDEO-selected" input catalog has a detection in the VIDEO catalog in at least one band.

In addition, a "fiducial" VIDEO band is selected for each source that is used for determining the source surface brightness profile model to be applied during the Tractor forced photometry. We preferentially select the VIDEO

\footnotetext{
${ }^{15}$ VIDEO source catalogs and images were obtained from the fifth data release available at http://horus.roe.ac.uk/vsa/.
} 
$K s$-band to be the fiducial band, but if the source is not detected at $K s$-band, we set the fiducial VIDEO band to the next closest filter in wavelength to the IRAC $3.6 \mu \mathrm{m}$ band that has a detection reported in the VIDEO source catalog. We note that, of 26 ALMA sources with optical-NIR counterparts in our catalog, 23 reside in the Ultra Deep tier of HSC ( $5 \sigma i$-band depth $\sim 27.2 \mathrm{mag}$ ) and three sources reside in the Deep HSC tier ( $5 \sigma i$-band depth $\sim 26.5 \mathrm{mag}$ ).

For each source in our VIDEO-selected input catalog, we extracted a cutout of the image in each of the 13 bands in our analysis with a half-width of $10^{\prime \prime}$. To account for spatial variations in the image properties, we measured the sky (rms) noise and the median background sky level in each image cutout using iterative sigma clipping with Photutils. ${ }^{16} \mathrm{We}$ then fit the source fluxes using the Tractor, which convolves the source surface brightness profile model with the image pointspread function and uses a maximum likelihood method to optimize the flux of each source in each band, holding the source position, shape, and image calibration properties fixed. We provide the resulting Tractor catalog of the forced photometry in Table 2 . We also compare the photometry using the Tractor with other surveys covering the same field to check consistency of our results. Here we utilize publicly available data release 8 of the UKIDSS-UDS survey. We refer our readers to Appendix B for the detailed discussion.

\subsection{Photometric Redshifts}

Photometric redshifts were estimated using the Easy and Accurate $z_{\text {phot }}$ from Yale (EAZY; Brammer et al. 2008, 2011) software. EAZY performs least square fitting with a linear combination of minimal template sets that can accommodate most of the variations in galaxy properties up to high redshift.

We use a default set SED templates available in EAZY. These templates adequately span the wavelength range to cover the 13 bands used in our analysis ( $4.5 \mu \mathrm{m}$ to $u$-band). EAZY provides a full probability distribution for the redshift values in the range $0<z_{\text {phot }}<6$. We select the photometric redshift of a given source at the value with the highest probability $\left(z_{\text {peak }}\right)$. The confidence intervals are selected such that the integrated probability distribution is equal to $95 \%$, corresponding to the EAZY output parameters 195 and $u 95$. Table 1 provides the photometric redshifts along with the $95 \%$ confidence limits of our sample.

We illustrate the EAZY photometric redshift fitting results for our sample galaxies in Figure 3. As shown in the green-shaded box in this figure, EAZY provides several diagnostic parameters to quantify the quality of the fit. The fitting results for the entire sample are shown in the Appendix B. We emphasize that our photometric redshifts are based on multiband forced photometry using the Tractor, a technique that has demonstrated improved photometric redshift accuracy compared to the use of positionmatched multiband photometric catalogs (Nyland et al. 2017). However, given the inherently dusty nature of our sources, which may cause deviations in their SEDs that are not well represented by the templates considered in this study, further verification of their redshifts will require a more in-depth SED analysis (to be presented in a forthcoming study) as well as future spectroscopic observations.

The rest-frame colors are also evaluated using best-fit SED template in EAZY. To estimate colors, we have used filters $U, V$,

\footnotetext{
$\overline{16}$ http://photutils.readthedocs.io/en/stable
}

and $J .{ }^{17}$ EAZY provides interpolated color indices for each filter of the rest-frame color, such that the colors can be calculated using the following formula; $U-V=-2.5 \times$ $\log \left(L_{U} / L_{V}\right)$, where $L_{U}$ and $L_{V}$ are the calculated $U$ - and $V$-band luminosities, respectively.

\subsection{The Final Catalog}

We present a catalog of 26 galaxies obtained by crossmatching the ALMA source catalog with optical-NIR observations (Section 2.1). The catalog contains 15 new, serendipitously discovered ALMA detections. From the remaining 11 known sources, nine were detected in the previous studies, and three correspond to a single bright Herschel source (Bussmann et al. 2015). We explain the properties of all previous detections later in this section. Figure 9 shows the four-band (ALMA band 6 or 7, Spitzer 3.6 and $4.5 \mu \mathrm{m}$, and VIDEO $K_{s}$ ) snapshots of the entire catalog. Table 1 summarizes the ALMA properties of our sample sources, our photometric redshifts, and any previously published redshift information. Throughout this article, we will be identifying sources by their IAU names given in column 1 of Table 1.

Nearly half of our targets $(11 / 26)$ were previously detected in the SIRTF Wide-area Infra Red Extragalactic Survey (SWIRE; Lonsdale et al. 2004), and photometric redshifts using the five-band SWIRE observations have been published (Rowan-Robinson et al. 2008, 2013). Another study that included a large number of our sample galaxies (13 out of 26) is that of Williams et al. (2009). These authors presented a $K$ band-selected galaxy catalog combining optical-mid-IR photometry from SWIRE, the Ultra Deep Survey (UDS) tier of the UKIRT Infrared Deep Sky Survey (UKIDSS; Lawrence et al. 2007), and the Subaru-XMM Deep Survey (SXDS; Sekiguchi \& SXDS Team 2004; Furusawa et al. 2008). They have used EAZY to estimate photometric redshifts on the basis of $B R i^{\prime} z^{\prime}$ $J K[3.6 \mu \mathrm{m}][4.5 \mu \mathrm{m}]$ photometry. Our photometric redshifts are in good agreement with previously published values (when available) within the margin of the uncertainties. We emphasize that the 13-band forced photometry presented here provides redshift estimates that are robust against the effects of source blending in the IRAC bands and utilize a large number of filters compared to the previous studies.

\section{Discussion}

\subsection{Flux and Redshift Distribution}

We show the flux distribution of our catalog in Figure 4; $57 \%$ of the sources have fluxes fainter than $1 \mathrm{mJy}$. The binomial uncertainty ${ }^{18}$ for each flux density bin is shown as the red line at the center of each bin. We plot the redshift distribution of the entire sample in Figure 5. Two separate histograms for different frequency bands (blue for band 6 and red for band 7) are shown. The median redshifts for bands 6 and 7 are $\langle z\rangle=2.72$ and 2.57 , respectively. We also compare the median redshift values from our work with those from other ALMA surveys. Our median redshift falls within the range of redshifts from other recent studies of SMGs.

Béthermin et al. (2015) showed that the median redshift of the sample of dusty galaxies depends significantly on the depth

\footnotetext{
${ }^{17} U$ and $V$ are standard Bessel filters and the $J$ filter follows the definition of Maunakea Consortium defined in Tokunaga et al. (2002).

18 The binomial uncertainty is defined as $\sigma_{n_{i}}=\sqrt{n_{i}\left(1-n_{i} / N\right)}$ where $n_{i}$ is the number of galaxies in bin $i$ and $N$ is the total number of galaxies.
} 
Table 2
Multiband Tractor Photometry

\begin{tabular}{|c|c|c|c|c|c|c|c|c|c|c|c|c|c|}
\hline $\begin{array}{l}\text { Source } \\
\text { (1) }\end{array}$ & $\begin{array}{l}F_{K s} \\
(2)\end{array}$ & $\begin{array}{l}F_{H} \\
(3)\end{array}$ & $\begin{array}{l}F_{J} \\
(4)\end{array}$ & $\begin{array}{l}F_{Y} \\
(5)\end{array}$ & $\begin{array}{l}F_{Z} \\
(6)\end{array}$ & $\begin{array}{l}F_{u^{\prime}} \\
(7)\end{array}$ & $\begin{array}{l}F_{g} \\
(8)\end{array}$ & $\begin{array}{l}F_{r} \\
(9)\end{array}$ & $\begin{array}{c}F_{i} \\
(10)\end{array}$ & $\begin{array}{c}F_{z} \\
(11)\end{array}$ & $\begin{array}{c}F_{y} \\
(12)\end{array}$ & $\begin{array}{c}F_{3.6 \mathrm{~mm}} \\
(13)\end{array}$ & $\begin{array}{c}F_{4.5 \mu \mathrm{m}} \\
(14)\end{array}$ \\
\hline $191313^{\mathrm{a}}$ & $0.37 \pm 0.06$ & $0.62 \pm 0.14$ & $0.35 \pm 0.03$ & $0.47 \pm 0.02$ & $0.28 \pm 0.01$ & $-0.03 \pm 0.01$ & $-0.04 \pm 0.01$ & $-0.07 \pm 0.01$ & $-0.06 \pm 0.02$ & $0.54 \pm 0.04$ & $0.38 \pm 0.08$ & $1.35 \pm 0.07$ & $0.81 \pm 0.10$ \\
\hline $309436^{\mathrm{a}}$ & $7.43 \pm 0.83$ & $6.48 \pm 0.22$ & $2.39 \pm 0.10$ & $1.38 \pm 0.07$ & $1.05 \pm 0.05$ & $0.04 \pm 0.02$ & $0.28 \pm 0.02$ & $0.90 \pm 0.03$ & $0.84 \pm 0.05$ & $1.06 \pm 0.08$ & $1.32 \pm 0.14$ & $7.00 \pm 0.14$ & $12.92 \pm 0.13$ \\
\hline $315617^{\mathrm{a}}$ & $0.51 \pm 0.07$ & $0.91 \pm 0.16$ & $0.81 \pm 0.04$ & $0.78 \pm 0.03$ & $0.55 \pm 0.02$ & $\ldots$ & $0.01 \pm 0.01$ & $0.20 \pm 0.01$ & $0.31 \pm 0.02$ & $0.42 \pm 0.04$ & $0.54 \pm 0.07$ & $-0.20 \pm 0.07$ & $-0.92 \pm 0.10$ \\
\hline 477360 & $6.83 \pm 0.72$ & $3.05 \pm 0.12$ & $1.23 \pm 0.07$ & $0.23 \pm 0.04$ & $-0.31 \pm 0.02$ & $-0.01 \pm 0.08$ & $-0.10 \pm 0.01$ & $-0.21 \pm 0.02$ & $-0.02 \pm 0.02$ & $-0.01 \pm 0.04$ & $0.36 \pm 0.08$ & $13.61 \pm 0.06$ & $24.10 \pm 0.09$ \\
\hline 814024 & $1.77 \pm 0.27$ & $0.93 \pm 0.06$ & $0.21 \pm 0.04$ & $0.05 \pm 0.02$ & $0.02 \pm 0.01$ & $-0.03 \pm 0.04$ & $0.01 \pm 0.01$ & $0.03 \pm 0.01$ & $0.01 \pm 0.01$ & $0.02 \pm 0.02$ & $0.10 \pm 0.04$ & $5.37 \pm 0.08$ & $7.12 \pm 0.11$ \\
\hline 842544 & $2.20 \pm 0.29$ & $1.68 \pm 0.07$ & $0.31 \pm 0.03$ & $0.18 \pm 0.02$ & $0.12 \pm 0.01$ & $0.01 \pm 0.04$ & $-0.01 \pm 0.01$ & $0.09 \pm 0.01$ & $0.05 \pm 0.01$ & $0.05 \pm 0.02$ & $-0.00 \pm 0.04$ & $10.84 \pm 0.07$ & $15.77 \pm 0.09$ \\
\hline 911805 & $11.12 \pm 1.12$ & $9.23 \pm 0.30$ & $3.89 \pm 0.14$ & $3.61 \pm 0.12$ & $3.04 \pm 0.10$ & $0.34 \pm 0.10$ & $1.40 \pm 0.04$ & $1.95 \pm 0.06$ & $2.54 \pm 0.08$ & $2.94 \pm 0.10$ & $3.04 \pm 0.14$ & $24.17 \pm 0.07$ & $32.00 \pm 0.09$ \\
\hline 932297 & $0.71 \pm 0.09$ & $0.59 \pm 0.06$ & $0.39 \pm 0.05$ & $0.51 \pm 0.09$ & $0.47 \pm 0.02$ & $0.00 \pm 0.04$ & $0.15 \pm 0.01$ & $0.46 \pm 0.02$ & $0.44 \pm 0.02$ & $0.52 \pm 0.03$ & $0.49 \pm 0.04$ & $-0.14 \pm 0.07$ & $0.17 \pm 0.08$ \\
\hline 935442 & $16.62 \pm 1.54$ & $10.80 \pm 0.33$ & $5.54 \pm 0.18$ & $4.02 \pm 0.12$ & $1.99 \pm 0.06$ & $0.11 \pm 0.06$ & $0.31 \pm 0.01$ & $0.57 \pm 0.02$ & $1.06 \pm 0.03$ & $1.83 \pm 0.06$ & $3.06 \pm 0.11$ & $26.82 \pm 0.04$ & $29.01 \pm 0.05$ \\
\hline 971686 & $0.44 \pm 0.07$ & $0.64 \pm 0.05$ & $0.35 \pm 0.04$ & $0.42 \pm 0.07$ & $0.29 \pm 0.01$ & $0.05 \pm 0.04$ & $0.30 \pm 0.01$ & $0.37 \pm 0.01$ & $0.31 \pm 0.01$ & $0.30 \pm 0.02$ & $0.27 \pm 0.03$ & $0.61 \pm 0.03$ & $1.07 \pm 0.05$ \\
\hline 978351 & $19.63 \pm 1.82$ & $10.49 \pm 0.33$ & $4.96 \pm 0.17$ & $2.16 \pm 0.08$ & $1.16 \pm 0.04$ & $0.11 \pm 0.09$ & $0.18 \pm 0.01$ & $0.44 \pm 0.02$ & $0.77 \pm 0.03$ & $1.17 \pm 0.05$ & $1.86 \pm 0.09$ & $39.81 \pm 0.04$ & $48.34 \pm 0.06$ \\
\hline 987090 & $7.04 \pm 0.67$ & $3.63 \pm 0.12$ & $0.73 \pm 0.05$ & $0.29 \pm 0.02$ & $0.18 \pm 0.01$ & $0.01 \pm 0.05$ & $-0.01 \pm 0.00$ & $0.01 \pm 0.01$ & $0.05 \pm 0.01$ & $0.13 \pm 0.02$ & $0.22 \pm 0.04$ & $15.35 \pm 0.04$ & $14.16 \pm 0.06$ \\
\hline 993676 & $14.72 \pm 1.35$ & $11.32 \pm 0.35$ & $6.05 \pm 0.19$ & $5.67 \pm 0.17$ & $3.98 \pm 0.12$ & $0.48 \pm 0.06$ & $0.83 \pm 0.03$ & $1.36 \pm 0.04$ & $2.06 \pm 0.06$ & $3.79 \pm 0.12$ & $4.79 \pm 0.15$ & $19.85 \pm 0.06$ & $21.56 \pm 0.09$ \\
\hline 998253 & $20.95 \pm 1.93$ & $12.57 \pm 0.39$ & $6.75 \pm 0.23$ & $5.56 \pm 0.17$ & $4.35 \pm 0.14$ & $0.48 \pm 0.10$ & $0.82 \pm 0.03$ & $1.28 \pm 0.04$ & $2.21 \pm 0.07$ & $4.30 \pm 0.13$ & $5.09 \pm 0.17$ & $37.95 \pm 0.08$ & $35.73 \pm 0.08$ \\
\hline 1002990 & $-0.06 \pm 0.06$ & $0.19 \pm 0.04$ & $0.21 \pm 0.03$ & $0.01 \pm 0.02$ & $0.13 \pm 0.03$ & $0.02 \pm 0.03$ & $0.06 \pm 0.00$ & $0.08 \pm 0.01$ & $0.10 \pm 0.01$ & $0.15 \pm 0.01$ & $0.16 \pm 0.03$ & $0.53 \pm 0.06$ & $0.32 \pm 0.09$ \\
\hline 1017745 & $19.10 \pm 1.77$ & $16.91 \pm 0.52$ & $11.27 \pm 0.35$ & $11.30 \pm 0.34$ & $9.29 \pm 0.28$ & $1.04 \pm 0.10$ & $2.00 \pm 0.06$ & $4.96 \pm 0.15$ & $7.11 \pm 0.21$ & $9.10 \pm 0.28$ & $10.68 \pm 0.33$ & $9.72 \pm 0.12$ & $9.64 \pm 0.12$ \\
\hline 1048801 & $13.33 \pm 1.27$ & $7.36 \pm 0.24$ & $3.83 \pm 0.14$ & $2.19 \pm 0.08$ & $1.56 \pm 0.05$ & $0.19 \pm 0.08$ & $0.47 \pm 0.02$ & $0.68 \pm 0.02$ & $1.06 \pm 0.04$ & $1.62 \pm 0.06$ & $1.88 \pm 0.08$ & $26.22 \pm 0.04$ & $32.26 \pm 0.07$ \\
\hline 1094072 & $1.42 \pm 0.24$ & $0.51 \pm 0.05$ & $0.22 \pm 0.04$ & $0.23 \pm 0.02$ & $0.21 \pm 0.01$ & $0.02 \pm 0.04$ & $0.02 \pm 0.00$ & $0.12 \pm 0.01$ & $0.12 \pm 0.01$ & $0.16 \pm 0.02$ & $0.17 \pm 0.03$ & $3.13 \pm 0.03$ & $3.35 \pm 0.06$ \\
\hline 1106738 & $0.75 \pm 0.11$ & $1.19 \pm 0.25$ & $0.86 \pm 0.06$ & $0.99 \pm 0.04$ & $0.94 \pm 0.03$ & $0.24 \pm 0.06$ & $0.68 \pm 0.02$ & $0.91 \pm 0.03$ & $0.96 \pm 0.03$ & $1.01 \pm 0.04$ & $1.00 \pm 0.07$ & $0.00 \pm 0.05$ & $-0.08 \pm 0.06$ \\
\hline 1266981 & $10.22 \pm 1.02$ & $5.58 \pm 0.19$ & $0.74 \pm 0.09$ & $0.56 \pm 0.04$ & $0.23 \pm 0.02$ & $0.02 \pm 0.07$ & $0.00 \pm 0.01$ & $0.07 \pm 0.01$ & $0.14 \pm 0.02$ & $0.20 \pm 0.03$ & $0.41 \pm 0.06$ & $21.25 \pm 0.05$ & $27.94 \pm 0.06$ \\
\hline 1302443 & $1.43 \pm 0.27$ & $0.57 \pm 0.06$ & $0.14 \pm 0.05$ & $-0.05 \pm 0.03$ & $-0.01 \pm 0.02$ & $-0.02 \pm 0.04$ & $0.02 \pm 0.01$ & $0.04 \pm 0.01$ & $0.03 \pm 0.01$ & $0.22 \pm 0.03$ & $-0.01 \pm 0.04$ & $3.96 \pm 0.13$ & $6.70 \pm 0.16$ \\
\hline 1302615 & $1.15 \pm 0.27$ & $0.30 \pm 0.05$ & $-0.04 \pm 0.05$ & $0.05 \pm 0.02$ & $-0.11 \pm 0.01$ & $-0.01 \pm 0.04$ & $0.00 \pm 0.01$ & $0.02 \pm 0.01$ & $0.01 \pm 0.01$ & $0.04 \pm 0.02$ & $0.06 \pm 0.04$ & $3.34 \pm 0.03$ & $4.86 \pm 0.05$ \\
\hline 1303410 & $1.87 \pm 0.28$ & $0.96 \pm 0.06$ & $0.31 \pm 0.04$ & $0.02 \pm 0.02$ & $0.11 \pm 0.01$ & $-0.01 \pm 0.04$ & $0.00 \pm 0.00$ & $-0.00 \pm 0.01$ & $0.01 \pm 0.01$ & $0.05 \pm 0.02$ & $-0.02 \pm 0.03$ & $4.82 \pm 0.04$ & $5.93 \pm 0.07$ \\
\hline 1304155 & $1.60 \pm 0.28$ & $0.88 \pm 0.07$ & $0.16 \pm 0.06$ & $0.12 \pm 0.04$ & $0.03 \pm 0.04$ & $-0.05 \pm 0.06$ & $0.01 \pm 0.01$ & $0.04 \pm 0.01$ & $0.03 \pm 0.01$ & $0.01 \pm 0.02$ & $0.08 \pm 0.04$ & $5.88 \pm 0.07$ & $8.44 \pm 0.09$ \\
\hline 1307256 & $0.61 \pm 0.26$ & $-0.14 \pm 0.10$ & $-0.23 \pm 0.12$ & $0.03 \pm 0.06$ & $0.07 \pm 0.05$ & $-0.02 \pm 0.04$ & $-0.01 \pm 0.00$ & $-0.02 \pm 0.01$ & $-0.01 \pm 0.01$ & $-0.03 \pm 0.02$ & $-0.04 \pm 0.03$ & $0.30 \pm 0.15$ & $3.02 \pm 0.11$ \\
\hline 1312163 & $0.86 \pm 0.28$ & $0.10 \pm 0.06$ & $-0.01 \pm 0.05$ & $0.10 \pm 0.03$ & $0.04 \pm 0.02$ & $-0.05 \pm 0.05$ & $0.00 \pm 0.01$ & $-0.03 \pm 0.01$ & $-0.02 \pm 0.01$ & $-0.03 \pm 0.02$ & $-0.09 \pm 0.04$ & $1.77 \pm 0.12$ & $2.99 \pm 0.13$ \\
\hline
\end{tabular}

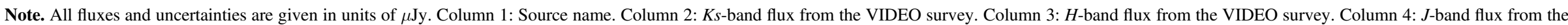

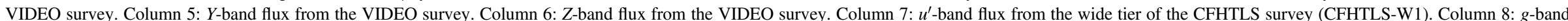

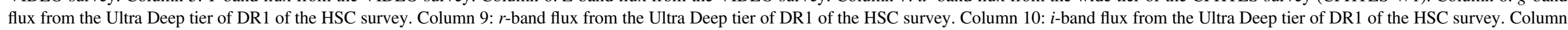

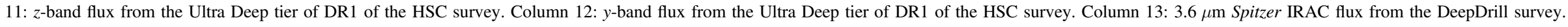
Column 14: $4.5 \mu \mathrm{m}$ Spitzer IRAC flux from the DeepDrill survey.

${ }^{\mathrm{a}}$ The CFHTLS flux is based on the deeper CFHTLS-D1 tier and the HSC flux is based on the shallower Deep tier. 
Object:1094072

Field: SXDF1100.230

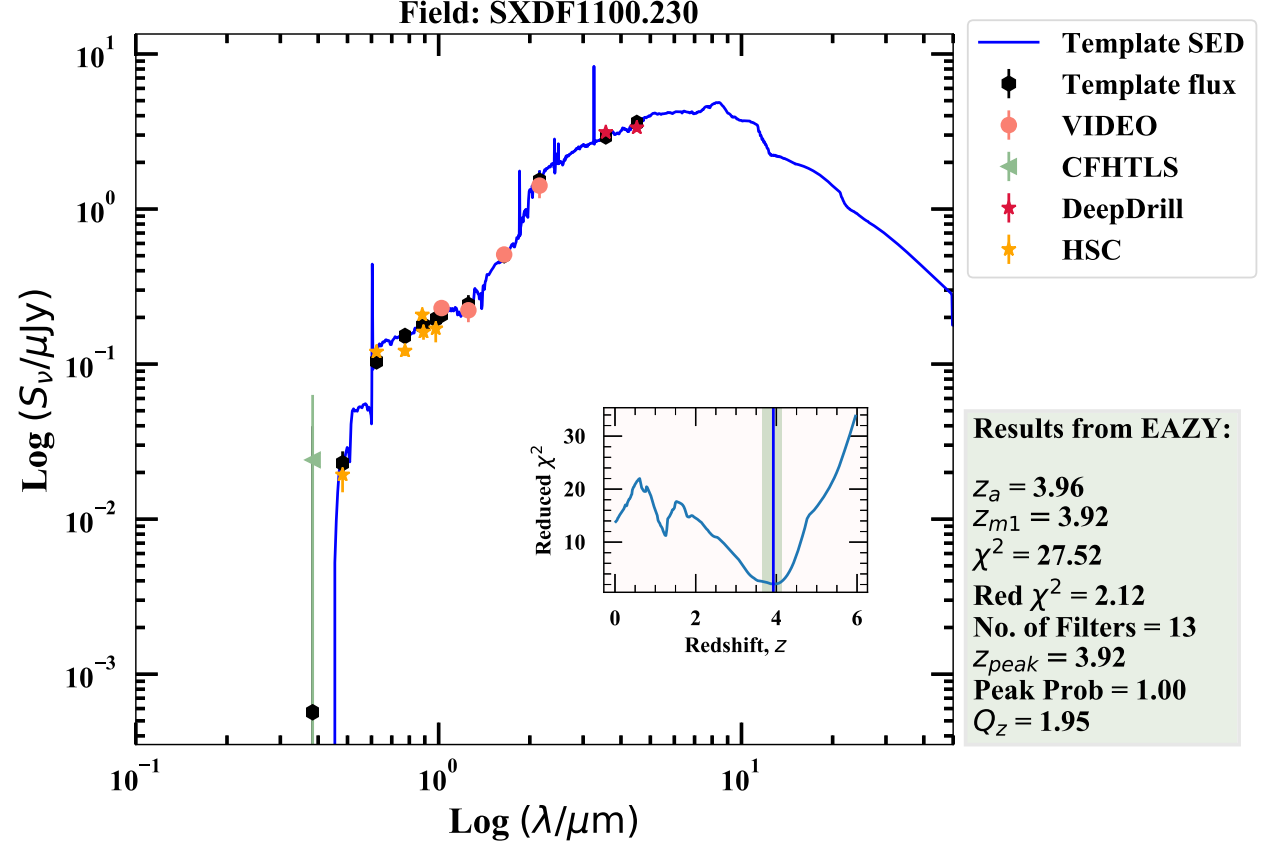

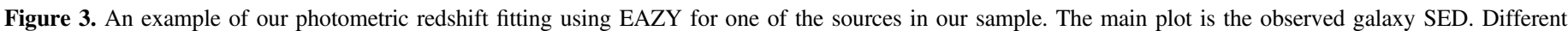

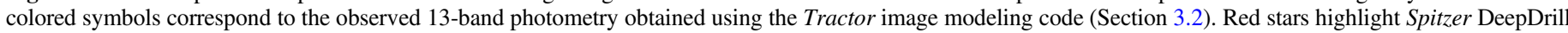

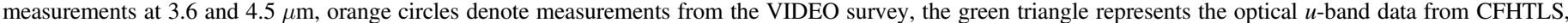

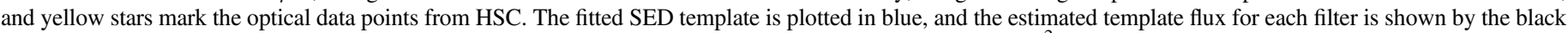

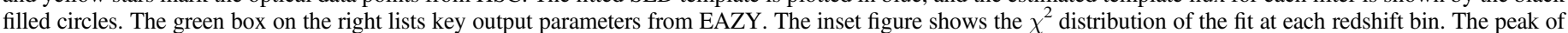
the redshift distribution after applying the prior is shown by the blue vertical line, and the light green regions are the $95 \%$ confidence intervals of the fit.

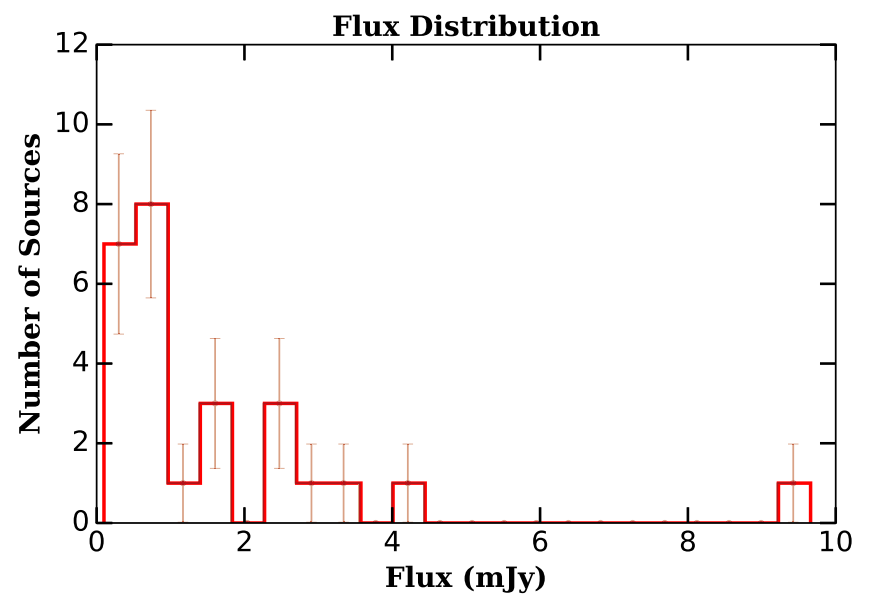

Figure 4. The flux distribution of our sample. The red line at the center of each bin is the binomial uncertainty. There are 15/26 galaxies (57\%) with fluxes fainter than $1 \mathrm{mJy}$. Except for one very bright galaxy $(S \sim 9.5 \mathrm{mJy})$, the rest of the bright sample has fluxes ranging from 1 to $5 \mathrm{mJy}$.

and the observing wavelength of the infrared surveys. They concluded that the median redshift increases with increasing wavelength up to $2 \mathrm{~mm}$ because of the negative $k$-correction. However, increasing the observing depth results in the detections of the lower-redshift, fainter sources. Hence, the observed variation in median values could just be an outcome of the varying depth of the surveys. The $1 \sigma \mathrm{rms}$ level in the $\sim 1.1 \mathrm{~mm}$ surveys by Aravena et al. (2016) and Dunlop et al. (2017) are $13 \mu \mathrm{Jy}_{\text {beam }^{-1}}$ and $30 \mu \mathrm{Jy}_{\text {beam }^{-1}}$, respectively. The median redshift values of their samples are also lower compared to the other studies $(\langle z\rangle=1.6$ and 2.15$)$. The survey by Brisbin et al. (2017) has a shallower depth of $150 \mu \mathrm{Jy}$ and, therefore, a higher

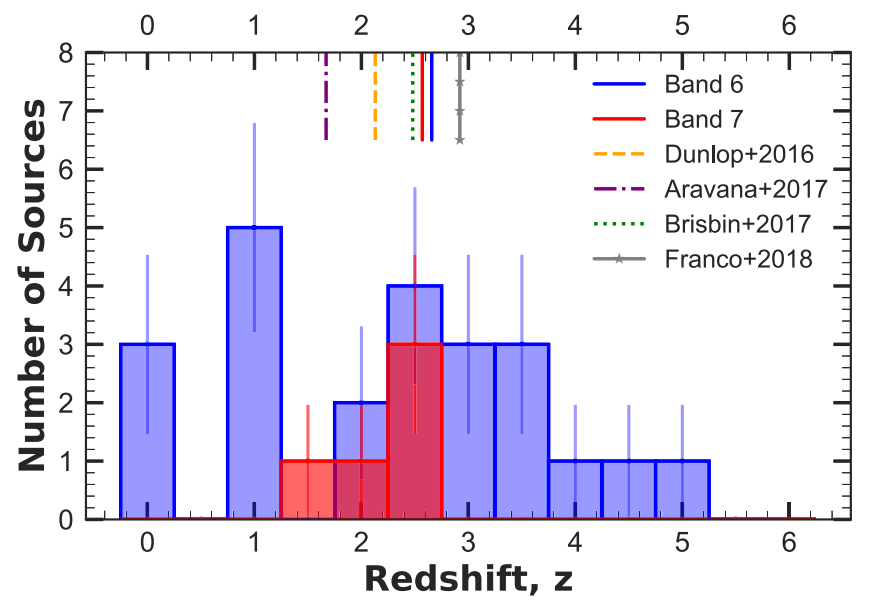

Figure 5. The redshift distribution of the faint SMGs. The photometric redshifts are evaluated using EAZY (Section 3.3). We show separate histograms for the ALMA frequency bands, 6 and 7. A line at the center of the bin shows the binomial uncertainty in each bin. The vertical lines at the top are the median redshifts for our sample as well as other faint SMG samples. Blue and red solid lines show the median values for bands 6 and 7 , respectively. Other studies included in the plot are as follows: Aravena et al. (2016), red dashed-dotted line; Dunlop et al. (2017), orange dashed line; Brisbin et al. (2017), green dotted line; and Franco et al. (2018), silver solid line with star symbols. The average depth for our band 6 sample is $110 \mu \mathrm{Jy}$. The median redshift and depth of band 6 data are consistent with the findings of Béthermin et al. (2015).

median redshift of 2.48. Continuing the trend, Franco et al. (2018) found a population at a median value of $\langle z\rangle=2.9$ on the basis of their survey with a sensitivity of $450 \mu \mathrm{Jy}$. In case of our work, the average depths for the band 6 and 7 are $110 \mu \mathrm{Jy}$ and $300 \mu \mathrm{Jy}$, respectively. Because we have only five galaxies in band 7 , we exclude those galaxies from any further analysis 
involving the median redshift. Even though we find a slightly larger median redshift for a depth shallower than do Brisbin et al. (2017), the redshifts and depths are comparable within the margin of errors. Therefore, our redshift distribution is consistent with other studies discussed in this section and the predictions by Béthermin et al. (2015).

\subsection{Previously Identified Galaxies}

As mentioned before, 11 ALMA detections in our catalog are not new. If these targets are DSFGs and unlensed, we have still included them in our catalog. With the availability of multiwavelength observations from SERVS and our robust 13-band photometry, we can better understand the nature of the previously identified galaxies and the overall population in general. We provide the references to the previous ALMA detection in Column 11 of Table 1 . Four of 11 galaxies were serendipitously detected in previous ALMA archival mining studies (J0216-0506, J0217-0454: Hatsukade et al. 2015; Fujimoto et al. 2016; J0217-0442: Fujimoto et al. 2016; J02170508: Ono et al. 2014).

Six of the 11 known galaxies are detected in the ALMA follow-up programs of the bright DSFGs. However, we still include targets in our analysis both the faint and bright SMGs belonging to the same category of dusty galaxies. Also, it would be interesting to observe differences in the multiwavelength properties of these two classes. Three of the six bright SMGs belong to a single Herschel source (J02190524.63, J0219-0524.77, and J0219-0524.84; Bussmann et al. 2015). The Herschel source is resolved into multiple components at the ALMA resolution. The alignment and similar mid-IR colors of these three galaxies indicate an overdense region. The ALMA flux densities of these targets are larger than those of the rest of our sample galaxies, but we will still include them to compare the physical properties. The remaining three galaxies (J0217-0508, J0217-0445, and J02170504) are selected from the AzTEC survey. These dusty, $z>3$ bright SMGs are thought to be the progenitors of low-redshift massive ellipticals (Ikarashi et al. 2015). They typically host a compact starburst in their centers, and their luminosities are comparable to nearby ultraluminous infrared galaxies.

The remaining galaxy J0226-0452 from the WMH5 field is a $z \sim 6$ UV-luminous Lyman Break galaxy (Willott et al. 2013). Some of the sources in our sample are part of the original ALMA program, which could lead to potential environmental biases in our results. However, 10 of 11 galaxies are either faint SMGs or selected from bright SMG surveys. Only one galaxy from our sample is the primary target of the original ALMA program, and no other galaxy has the same redshift as the primary target (where the redshift of the primary target is known). Given the small amount of overlap between the ALMA primary targets and our faint SMGs, we do not believe that our sample is significantly affected by environmental biases.

Spectroscopic redshifts are available for three targets (J02260452, J0216-0506, and J0217-0454) taken from different studies, and the references are given in the Table 1. Target J0216-0506 is detected in most of the studies mentioned above, and Seko et al. (2016a) have discussed the spectroscopic redshift and interstellar medium properties of that galaxy. It is a member of the sample of $z \sim 1.4$ star-forming main-sequence galaxies. CO emission studies with ALMA have indicated

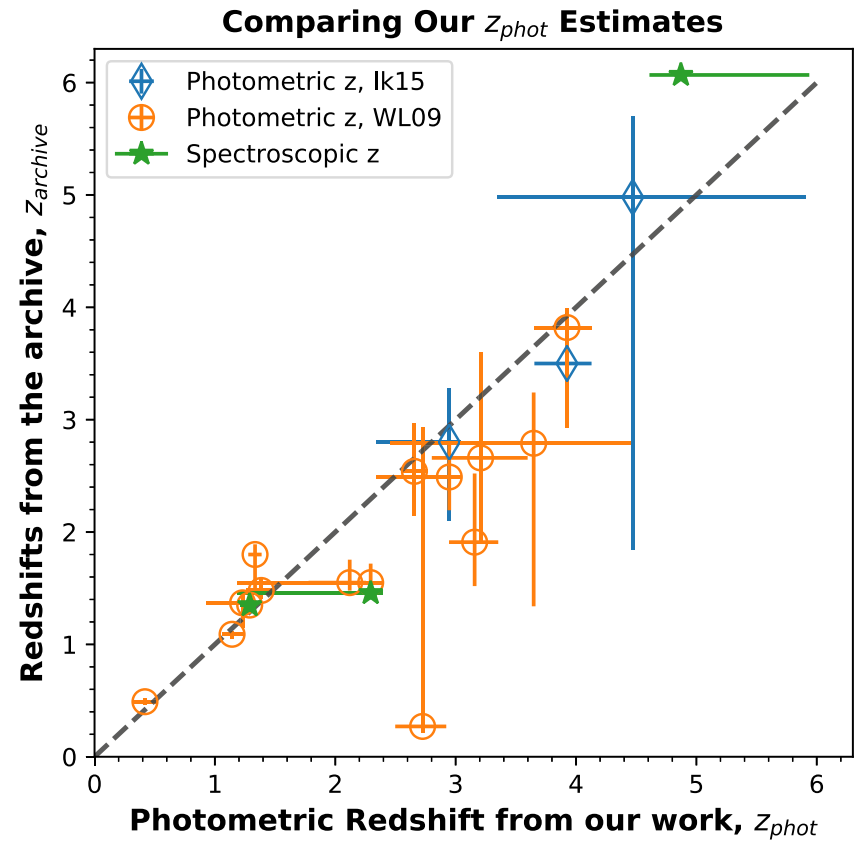

Figure 6. A comparison of the photometric redshifts evaluated in this work with redshift estimates from previous studies. A total of 18 of 26 sources have either photometric or spectroscopic redshifts available in the literature. The green points correspond to sources with spectroscopic redshifts. The orange and blue points have photometric redshift estimates published by Williams et al. (2009) and Ikarashi et al. (2015), respectively. The uncertainties from our work are smaller than those in previous studies, highlighting our improved photometry through the use of the Tractor image modeling software.

larger molecular gas fractions and gas-to-dust ratios than in local galaxies. Banerji et al. (2011) have identified the target J0217-0454 as a Submillimeter Faint Radio Galaxy, which is similar to bright SMGs but has hotter dust temperatures. Its spectroscopic redshift $(z=1.456)$ was measured using [OII] 3727 emission line. In all cases, the spectroscopic redshifts are well within the $95 \%$ confidence interval of our photometric redshifts.

Figure 6 compares the performance of our redshift estimation with archival results. The purpose of this comparison is to conduct a consistency check on the forced photometry technique used in this work. The overall uncertainties on the photo- $z$ estimates are small in most cases. The values are in very good agreement at lower redshifts, $z<2.5$. However, at higher redshifts, we see an increased scatter in the redshift agreement. This could be due to large uncertainties in the photometric measurements, differences in the filters used for the photo- $z$ estimation, and inherent limitations of the SED templates. Our photometry includes $u$-band data, which is lacking in the previous studies. Also, different methods of source deblending in the Spitzer bands could lead to differences in the photometry. This issue can be resolved using spectroscopic data.

\subsection{Optical-NIR Triple Color Selection}

Chen et al. (2016b) have devised a selection technique to identify bright as well as faint SMGs using a training data set from the UKIDSS-UDS field. The method is called optical-IR triple-color selection (OIRTC) and uses three optical-NIR colors: $z-K_{s}, K_{s}-$ [3.6], and [3.6]-[4.5]. The color cuts are 


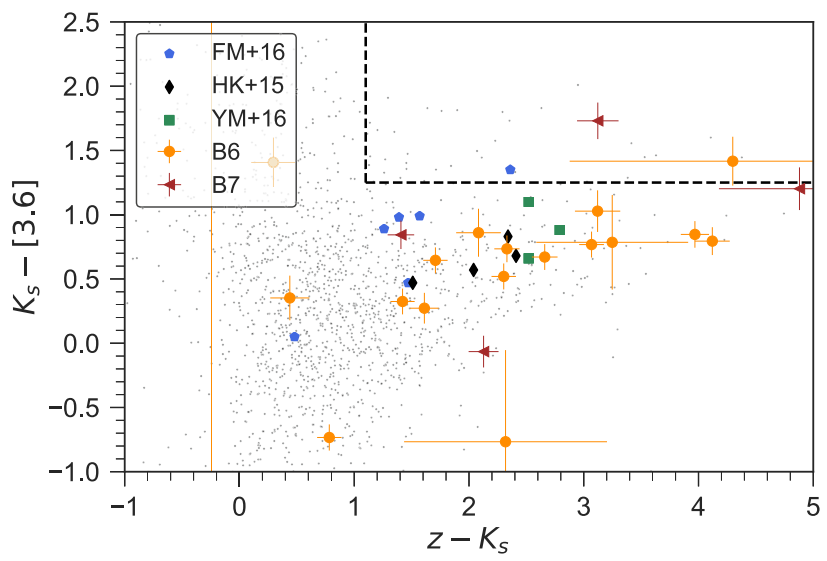

(a)

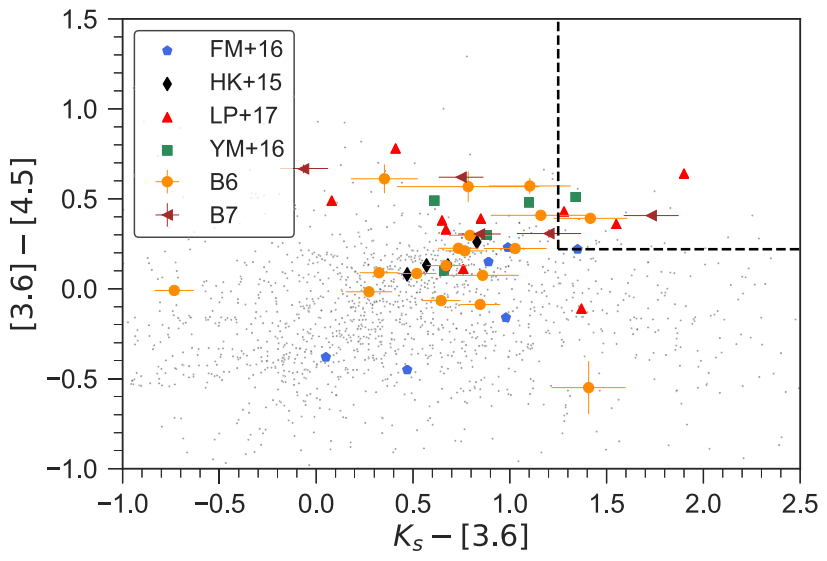

(b)

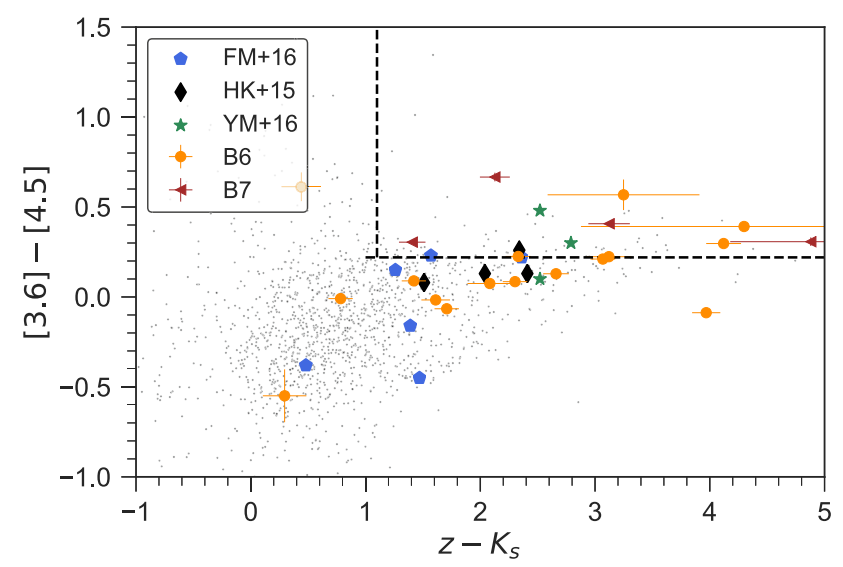

(c)

Figure 7. The plot shows the SMG OIRTC color selection criteria defined in Chen et al. (2016b). Each subfigure is a color-color plot of the combination of $z, K_{s}$, [3.6], and [4.5] filters. Bright SMGs tend to occupy the reddest part of the color space because of the presence of dust. The black dotted line in each subfigure is a color cutoff estimated by Chen et al. (2016b) using an ALMA sample of bright SMGs. The fraction of SMGs in the population redder than the color threshold is at least 5\%. The yellow filled circles (B6) and brown horizontal triangles (B7) represent band 6 and 7 sources in our sample, respectively. Other faint SMG samples are shown as follows: YM+16 (green squares), Yamaguchi et al. (2016); HK+15 (black diamonds), Hatsukade et al. (2015); FM+16 (blue pentagons), Fujimoto et al. (2016); and LP+17 (red vertical triangles), Laporte et al. (2017). The light-gray symbols in the background show the field non-SMG galaxies found within all ALMA pointings. Our sample occupies a bluer color space than does the OIRTC selection cutoff mainly because of the mailKs - [3.6] $\mu \mathrm{m}$ color. This shows that the optical-NIR color properties of our faint SMG population are different from the bright SMGs.

defined such that the mean SMG fraction redder than the color threshold is at least $5 \%$. The training sample contains ALMA and $850 \mu \mathrm{m}$ SCUBA-2 observations of bright SMGs. By combining radio and optical-NIR selection techniques, their identification is about 83\% complete. Chen et al. (2016a) have utilized this technique to identify faint SMGs with expected fluxes, $S_{850 \mu \mathrm{m}}<2 \mathrm{mJy}$.

Here, we compare the optical-IR colors of our faint SMG sample with the color selection method described in Chen et al. (2016b). Figure 7 illustrates the OIRTC color selection, where different colored symbols represent our sample along with faint SMG samples of Hatsukade et al. (2015), Fujimoto et al. (2016), Yamaguchi et al. (2016), and Laporte et al. (2017). A comparison with other samples will allow us to evaluate the significance of our results and also to check the consistency between different methods adopted in all of the studies. We see that almost our entire sample occupies the color space outside the selection cuts defined by the OIRTC technique. Furthermore, the galaxies from the comparison samples also lie mostly outside the OIRTC color selection. The light-gray symbols in Figure 7 are the non-SMG field galaxies found within the search radius of all the ALMA pointings used in our study. These field galaxies will allow us to check whether the colors of faint SMGs are systematically redder.

We observe that $75 \%$ of faint SMGs satisfy the $z-K_{s}$ color criterion of having redder colors than the OIRTC $z-K_{s}$ color criterion, whereas only $47 \%$ and $18 \%$ of the faint SMG population satisfy the OIRTC [3.6] $\mu \mathrm{m}-[4.5] \mu \mathrm{m}$ and $K_{s}-$ [3.6] $\mu \mathrm{m}$ color criteria, respectively. Laporte et al. (2017) and Cowie et al. (2018) have also analyzed the OIRTC criteria for their sample sources. Both studies found that majority of their samples do not satisfy the OIRTC $K_{s}-$ [3.6] $\mu \mathrm{m}$ color cut. A few factors could be responsible for the differences. The primary extrinsic factor is the variations in the photometry techniques used to obtain colors. Laporte et al. (2017) pointed out that the disagreement of the $K_{s}-$ [3.6] colors between their and Chen et al. (2016b) sample was mainly due to the differences in the Spitzer IRAC aperture correction methods. When the OIRTC color criteria were recalibrated for their field galaxies, the new color cuts were $80 \%$ complete. The Tractor forced photometry presented in our work takes the point-spread function of each band into account, thus removing the need for aperture corrections. Nevertheless, we still 


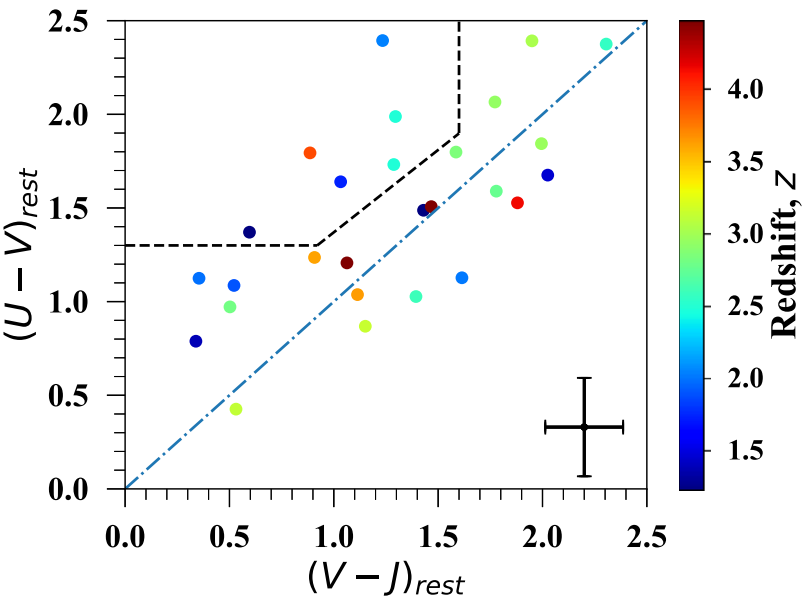

Figure 8. $U V J$ color selection diagram. The symbols are color-coded according to their photometric redshifts, and the color bar is shown on the right. Five of the 26 galaxies lie in the quiescent galaxy clump. The blue dotted line is a proportionality line and is plotted to identify the diagonal track occupied by the star-forming galaxies. The black error bar in the bottom-right corner shows the average uncertainties in the color estimation.

find a significant fraction of the sample occupying bluer Ks-[3.6] colors, which may be due to the differences in the intrinsic properties of the faint and bright SMGs. Hence, additional checks are needed before directly applying color cuts from the other studies. It would be useful to perform future studies which combine all the different faint SMGs and provide consistent photometry for all the comparison samples.

As mentioned above, the other reason could be the intrinsic differences in the SEDs of bright and faint SMGs. The OIRTC color selection method is trained using bright SMGs, and the faint population in Chen et al. (2016a) does not have confirmed interferometric detections. Hence, the technique is targeting faint galaxies having SEDs similar to the bright SMGs. To further support this, Hatsukade et al. (2015) found bluer colors for their sample of faint SMGs. Therefore, additional study is needed, including spectroscopic follow-up, in order to better understand the colors of faint SMGs.

\subsection{The UVJ Color-color Plot}

At higher redshifts, where it is difficult to classify galaxies on the basis of their morphologies, classifications via rest-frame broadband colors have proven to be useful. Star-forming and quiescent galaxies show a bimodality in rest-frame colors up to $z \sim 2.5$ in the $U V J$ color-color diagram (Labbé et al. 2005; Wuyts et al. 2007; Williams et al. 2009; Brammer et al. 2011; Whitaker et al. 2011; Patel et al. 2011). The quiescent galaxies form a red clump called the "red sequence," and star-forming galaxies fall on a diagonal line. The redder galaxies on the starforming main sequence have higher dust extinction. The $U-V$ and $V-J$ colors of the DSFGs are both reddened by dust extinction. However, in the case of quiescent galaxies, only the $U-V$ colors are reddened as a result of the Balmar/ $4000 \AA$ break, and the $V-J$ colors are bluer as compared to the $U-V$ (Chen et al. 2016b). Therefore, the $V-J$ color is a good proxy for dust extinction, and the two colors can separate DSFGs and red sequence quiescent galaxies up to $z \sim 2.5$.

Figure 8 shows the rest-frame $U V J$ color diagram for our sample. The rest-frame colors are estimated using EAZY, as explained in Section 3.3. To estimate the uncertainties in the $U$,
Table 3

Results of Stacking $>5 \sigma$ ALMA Objects That were Undetected in the IRAC Bands

\begin{tabular}{lccr}
\hline \hline Stack & Number & $3.6 \mu \mathrm{m}$ & $4.5 \mu \mathrm{m}$ \\
& & $\mathrm{AB}$ mag & $\mathrm{AB}$ mag \\
\hline All undetected & 60 & $>26.1$ & $>26.1$ \\
$>1 \mathrm{mJy}$ & 44 & $>25.8$ & $>25.9$ \\
$<1 \mathrm{mJy}$ & 16 & $>25.5$ & 25.3 \\
\hline
\end{tabular}

$V$, and $J$ bands, we find the two nearest filters for each band and add their errors in quadrature. Then, we calculate the uncertainties of the rest-frame colors using a simple error propagation rule. Here, we consider only measurement errors and exclude the errors in the SED templates and fitting.

The quiescent galaxy color cuts are taken from Williams et al. (2009). The color selection is defined as follows:

$$
\begin{gathered}
U-V>1.3 \\
V-J<1.6 \\
U-V>0.88 \times(V-J)+0.49 .
\end{gathered}
$$

The third criterion has a small dependence on redshift, and we used the equation for the redshift bin $1<z<2$. Five of 26 galaxies are located within the quiescent box, and the rest of the sample has colors similar to those of the star-forming galaxies. When we compare the bicolor sequence from Williams et al. (2009), we see that our star-forming sample occupies redder $U-V$ and $V-J$ colors indicating the dusty and high-redshift nature of our sample.

\subsection{Stacking of Undetected ALMA Sources}

We used stacking to investigate the nature of the faint population that was not individually detected in our photometric catalog. Because the rate of contamination of the ALMA catalog by noise fluctuations is expected to be very small above $5 \sigma$, we stacked the DeepDrill IRAC data at the positions of the $>5 \sigma$ sources $(60 / 176)$. We divided our sample into sources that were bright $(>1 \mathrm{mJy})$, or faint $(<1 \mathrm{mJy})$ We only detected $>3 \sigma$ emission in the IRAC $4.5 \mu \mathrm{m}$ channel stack of the faint sources (Table 3$)$ at the level of $0.34 \mu \mathrm{Jy}(A B=25.3)$. This indicates that the majority of the undetected population is extremely faint in the IRAC bands $(A B \gtrsim 25)$, and is either of low stellar mass $\left(\lesssim 10^{10} M_{\odot}\right.$ at $\left.z=2\right)$ and/or highly reddened.

\section{Summary and Conclusions}

In this article, we have successfully demonstrated the use of archival ALMA observations to search for faint SMGs. The ALMA detections in the XMM-LSS field greater than $3.9 \sigma$ were cross-matched with the deep optical-NIR observations taken from SERVS/DeepDrill, VIDEO, CFHTLS, and HSC. We identify 26 sources with NIR counterparts, 15 of which are newly identified faint SMGs. To further investigate the nature of this cosmologically important population, we have analyzed the basic properties of our sample. Of the total 26 sources, there are 16 faint SMGs $\left(S_{1.1 \mathrm{~mm}}<1 \mathrm{mJy}\right)$ with a median flux of $0.57 \mathrm{mJy}$ and 10 bright SMGs with a median flux of $2.44 \mathrm{mJy}$. Robust 13-band forced photometry using the Tractor image modeling code is available for our entire catalog.

The resulting photometric measurements were used to estimate photometric redshifts and rest-frame colors. Our 
sources have redshifts in the range of $0.4<z_{\text {phot }}<5.3$, with a median photometric redshifts of $\langle z\rangle=2.72$ and 2.57 for band 6 and 7 , respectively. The median redshift and the average depth of our search are in good agreement with the predictions given in Béthermin et al. (2015).

We performed an optical-NIR triple-color selection that showed that most of our sample galaxies have bluer colors than the redder bright SMGs. Different color properties of faint SMGs could indicate different physical properties compared to their brighter counterparts. On the basis of the rest-frame $U V J$ colors, we found that most of the galaxies in our sample form the star-forming diagonal track on the $U V J$ diagram. Their colors are consistent with star-forming main-sequence galaxies.

ALMA has made the discovery of this population of DSFGs possible. We will continue mining publicly available ALMA archival observations and expand our search to the remaining five SERVS/DeepDrill fields to find a large sample of faint SMGs. We will include robust optical-NIR photometry along with far-IR Herschel observations to perform SED modeling of all the targets and estimate stellar masses, star formation rates, and other physical properties. Pilot studies (including this work) are unveiling the nature of the faint SMGs that dominate the CIB. Future large-scale surveys are essential to understanding the role of these galaxies in shaping galaxy evolution.

The National Radio Astronomy Observatory is a facility of the National Science Foundation (NSF) operated under cooperative agreement by Associated Universities, Inc. Support for this work was provided by the NSF through the Grote Reber Fellowship Program administered by Associated Universities, Inc./National Radio Astronomy Observatory. This paper makes use of the following ALMA data: ADS/JAO.ALMA \#2011.0. 00115.S, \#2011.0.00648.S, \#2011.0.00539.S, \#2012.1.00326.S, \#2012.1.00374.S, \#2012.1.00934.S, \#2013.1.00815.S, \#2013. A.00021.S, \#2015.1.01105.S. ALMA is a partnership of ESO (representing its member states), NSF (USA), and NINS (Japan), together with NRC (Canada) and NSC and ASIAA (Taiwan) and KASI (Republic of Korea), in cooperation with the Republic of Chile. The Joint ALMA Observatory is operated by ESO, AUI/ NRAO, and NAOJ. This work is based on observations made with the Spitzer Space Telescope, which is operated by the Jet Propulsion Laboratory, California Institute of Technology under a contract with NASA. K.N. acknowledges support provided by the grant associated with Spitzer proposal 11086. J.A. gratefully acknowledges support from the Science and Technology Foundation (FCT, Portugal) through the research grants No. PTDC/FISAST/2194/2012, No. PTDC/FIS-AST/29245/2017, No. UID/ FIS/04434/2013, and No. UID/FIS/04434/2019.

The authors have made use of ASTROPY, a communitydeveloped core PYTHON package for Astronomy Astropy Collaboration et al. (2013). We also used MONTAGE, which is funded by the National Science Foundation under grant No. ACI-1440620, and was previously funded by the National
Aeronautics and Space Administration's Earth Science Technology Office, Computation Technologies Project, under Cooperative Agreement No. NCC5-626 between NASA and the California Institute of Technology. This research has made use of the NASA/IPAC Extragalactic Database (NED), which is operated by the Jet Propulsion Laboratory, California Institute of Technology, under contract with the National Aeronautics and Space Administration.

Facilities: ALMA, Spitzer, CFHT, VISTA, Subaru.

\section{Appendix A The ALMA Archival Projects}

Nine ALMA projects satisfied our selection criteria given above and were publicly available. These projects were undertaken for entirely different science goals. The data are taken from Ouchi et al. (2013), who observed a giant starburst galaxy at redshift $z \sim 7$, called Himiko, at band 6 . The total integration time was about $3 \mathrm{hr}$, reaching noise levels $\sim 19 \mu \mathrm{Jy}_{\text {beam }^{-1}}$. Other deep maps were taken from ALMA 2013.1.00815.S (Willott et al. 2015). They have investigated continuum dust emission and [CII] line detections for three more $U V$-luminous galaxies similar to what Himiko found at $z \sim 6$. The total time on the source is about an hour and a half with rms noise levels of $18 \mu \mathrm{Jy}_{\mathrm{beam}^{-1}}$. The ALMA 2012.1.00934.S [PI: Phillip Best] covers four maps in the XMM-field to study the star formation activity in moderately star-forming galaxies at $z \sim 2.53$ using $\mathrm{CO}$ molecular line emission. Ikarashi et al. (2015) have observed 30 potential high- $z$ SMG candidates selected from the AzTEC survey. These galaxies are highly likely to be at $z>3$. This project, ALMA 2012.1.00326.S, has 4 minutes on-source time with rms levels between 135 and $65 \mu \mathrm{Jy}_{\text {beam }^{-1}}$. A 100 minute observation was undertaken by Inoue et al. (2016) [ALMA 2012.1.00374.S, PI: Kazuaki Ota] to study the state of the epoch of reionization and star formation activity at $z \sim 7$ using spectroscopically confirmed galaxies at that redshift. The same target was observed for another 75 minutes in the project ALMA 2013.A.00021.S to improve the sensitivity of [CII] line detection. The data from ALMA 2011.0.00648.S is taken to study interstellar medium of 20 star-forming galaxies at $z \sim 1.4$ (Seko et al. 2016a, 2016b). The typical rms noise levels range from $60 \sim 100 \mu \mathrm{Jy}_{\text {beam }}{ }^{-1}$ with 10 minutes of the on-source time. Pentericci et al. (2016) studied a very highredshift galaxy $(z \sim 7)$ to understand the role of high- $z$ galaxies in the epoch of reionization. The target was observed for 35 minutes which resulted in $20 \mu \mathrm{Jy}$ noise level. The project 2011.0.00539.S was used to study the ALMA properties of the lensed, bright SMGs selected from Herschel. The time on each map is about a minute, with typical rms noise levels of $250 \mu \mathrm{Jy}_{\text {beam }^{-1}}$. This ALMA project is the only band 7 observations we have used in our search. 


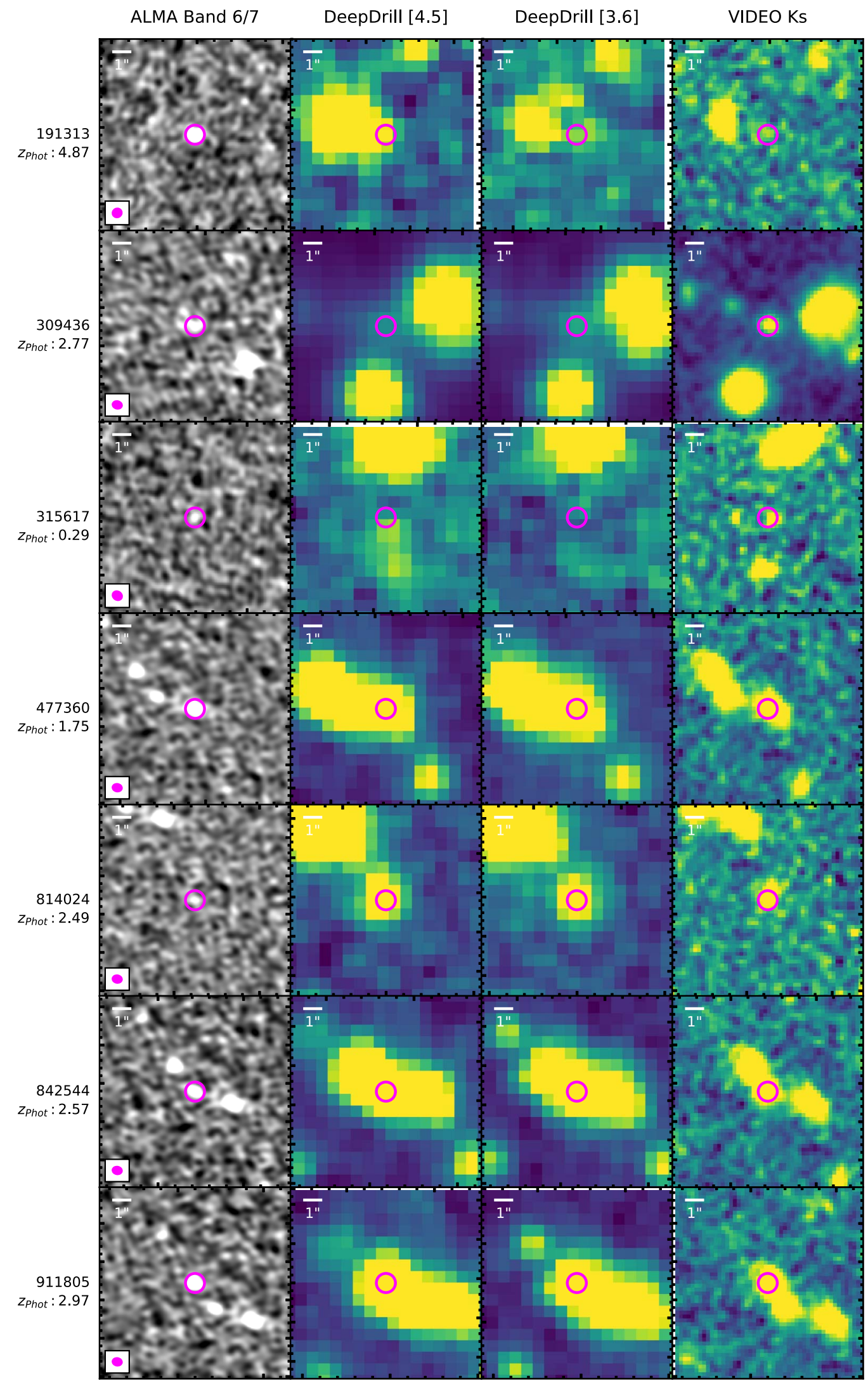

Figure 9. Image snapshots of our entire sample in four bands. The grayscale image on the left is the ALMA band 6/7 image obtained from the archive. The rest are the Spitzer $4.5 \mu \mathrm{m}$, Spitzer $3.6 \mu \mathrm{m}$, and VIDEO $\mathrm{K}_{s}$ image left to right. The magenta circle in each image indicates the position of the ALMA source. Our photometric redshift estimates are also given on the left. Each cutout has a size of $10^{\prime \prime} \times 10^{\prime \prime}$. All the sources are available in the figure set.

(The complete figure set (4 images) is available.) 


\section{Appendix B Photometry Consistency Check}

As we compare colors of the faint SMGs with the selection criteria specified by Chen et al. (2016b), it is essential to perform a consistency check within both the data catalogs. Our Tractor method utilizes the VIDEO catalog, whose photometry is in the $\mathrm{AB}$ magnitude system. The sample of Chen et al. (2016b) is taken from UKIDSS Ultra Deep Survey (UDS) data release 8 (DR8). We compare $J, H$, and $K$ bands from the publicly available DR $8^{19}$ with the VIDEO catalog in Figure 10. The data points plotted here are the field galaxies lying within the ALMA pointings used in our search. The positions of those objects were crossmatched with the publicly available UDS DR8 catalog. We show Petrosian magnitudes for both the catalogs in the $\mathrm{AB}$ system. Overall, there is a good level of agreement between our photometry and the UDS photometry. We can see a small offset between the $K$ filter used in the UKIDSS-UDS surveys and the $K_{s}$ filter used in the VIDEO survey. The offset is mainly due to the differences in the filter response functions of $K$ and $K_{s}$.
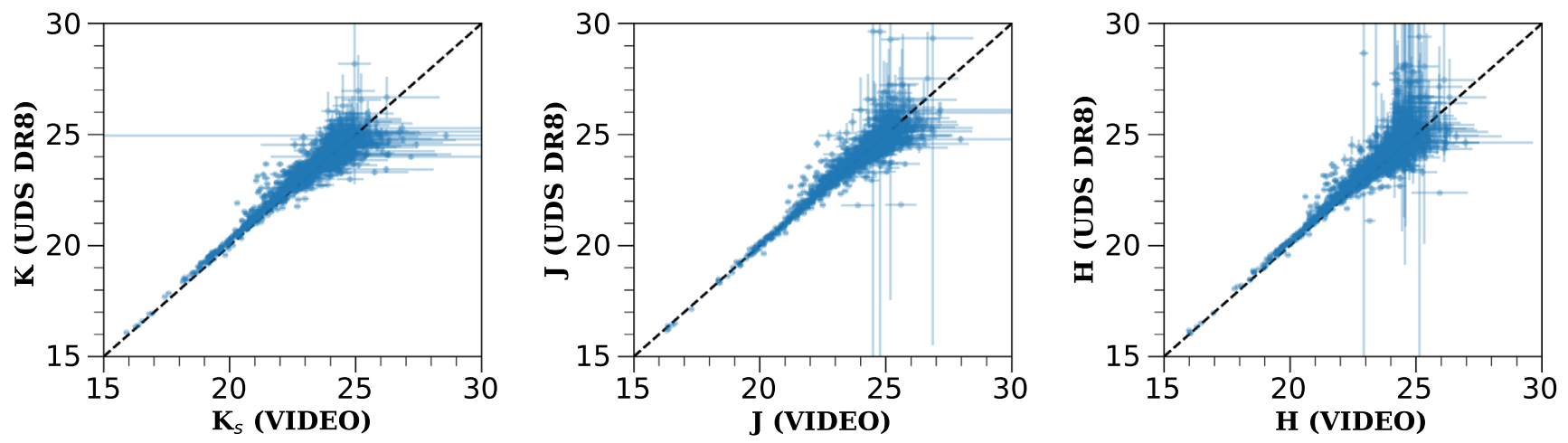

Figure 10. The plot shows a comparison of the photometry from our work and the publicly available UKIDSS-UDS DR8 catalog. Our photometry is derived from the VIDEO catalog (See Section 3.2). We show Petrosian magnitudes in the AB system for both catalogs. The black dashed line is the equality line. We see overall good agreement between both the observations. A slight offset in the $K$ band is mainly due to the differences in the filter response function.

\footnotetext{
${ }^{19}$ We used the following link to access the UDS photometry. http://wsa.roe. ac.uk:8080/wsa/crossID_form.jsp.
} 
191313

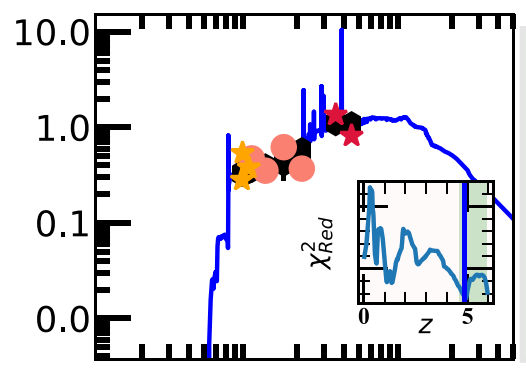

315617

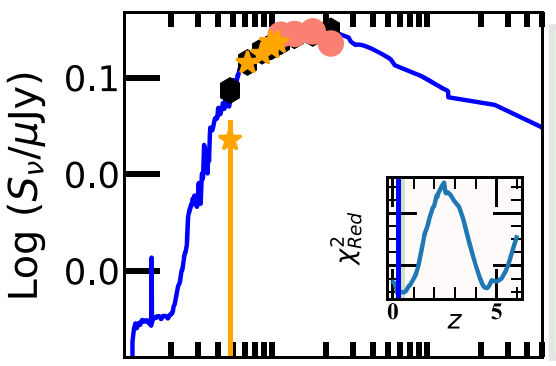

814024

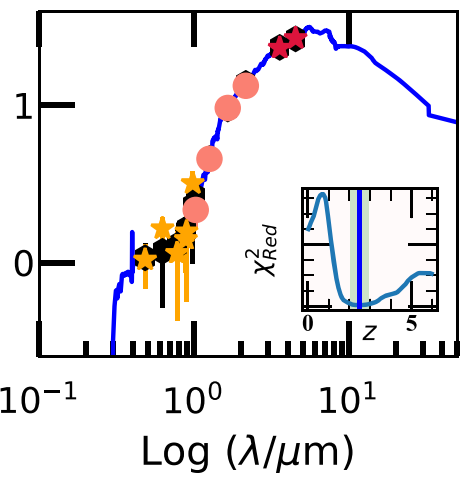

309436

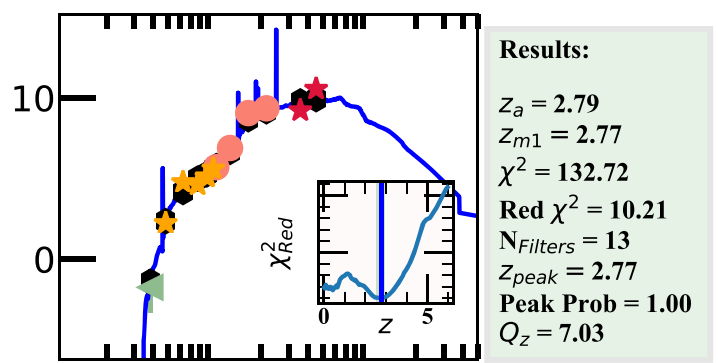

477360

Results:

$Z_{a}=0.23$

$z_{m 1}=0.29$

$\chi^{2}=\mathbf{1 1 7 . 5 6}$

Red $\chi^{2}=11.76$

$\mathbf{N}_{\text {Filters }}=\mathbf{1 0}$

$z_{\text {peak }}=0.29$

Peak Prob $=1.00$

$Q_{z}=7.60$

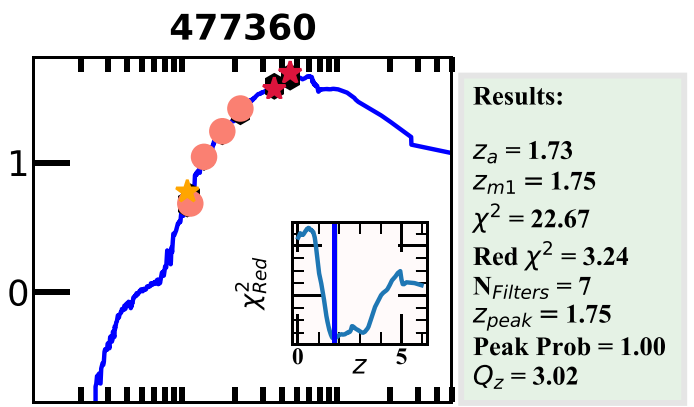

842544
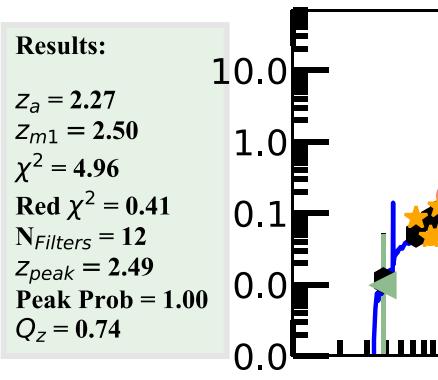

11

$10^{-1}$

$$
\begin{aligned}
& 10^{0} \quad 10^{1} \\
& \log (\lambda / \mu \mathrm{m})
\end{aligned}
$$

Figure 11. The photometric redshift fitting of the entire sample. Different colored symbols correspond to the observed 13-band photometry, which is obtained using the Tractor image modeling code (Section 3.2). Red stars highlight Spitzer DeepDrill measurements at 3.6 and $4.5 \mu \mathrm{m}$, orange circles denote measurements from the VIDEO survey, the green triangle represents the optical $u$-band data from CFHTLS, and the yellow stars mark the optical data points from HSC. The blue solid line is the actual SED fit, and the black filled circles show the estimated template flux for each filter. The green box on the right lists key output parameters from EAZY. The inset figure shows the $\chi^{2}$ distribution of the fit at each redshift bin. The blue vertical line shows the peak of the redshift distribution after applying the prior, and the light green regions are the $95 \%$ confidence intervals of the fit. All the sources are available in the Figure Set.

(The complete figure set (5 images) is available.)

\section{Appendix C}

\section{List of Continuum Maps from the ALMA Archive}

Table 4 provides a list of continuum maps used in the search of the faint SMG population. There are 75 individual pointings used in our work, and the sky position, date of observation, project ID, central frequency of observation, clean beam size, and rms noise levels are given in the article. 
Table 4

Details of the Archival Observations and Individual Pointings

\begin{tabular}{|c|c|c|c|c|c|c|c|c|c|}
\hline Index & Map & $\begin{array}{c}\text { R.A. } \\
\text { (J2000) }\end{array}$ & $\begin{array}{c}\text { Decl. } \\
\text { (J2000) }\end{array}$ & $\begin{array}{c}\text { Date of } \\
\text { Observation } \\
\quad \text { (yyyy } \\
-\mathrm{mm}-\mathrm{dd})\end{array}$ & Project ID & $\begin{array}{c}\nu_{\mathrm{obs}} \\
(\mathrm{GHz})\end{array}$ & $\begin{array}{l}\lambda_{\text {obs }} \\
(\mathrm{mm})\end{array}$ & $\begin{array}{l}\text { Resolving } \\
\text { Beam } \\
\left("{ }^{\prime \prime}\right)\end{array}$ & $\begin{array}{c}\text { rms Noise } \\
\left(\mu \mathrm{Jy} \mathrm{beam}^{-1}\right)\end{array}$ \\
\hline 0 & CLM1 & $02^{\mathrm{h}} 28^{\mathrm{m}} 02^{\mathrm{s}} .970$ & $-04^{\mathrm{d}} 16^{\mathrm{m}} 18^{\mathrm{s}} .30$ & 2014 Jun 16 & 2013.1.00815.S & 258 & 1.16 & $0.53 \times 0.46$ & 15.42 \\
\hline 1 & $\begin{array}{c}\text { HiZELS-UDS- } \\
\text { NBK-1147 }\end{array}$ & $02^{\mathrm{h}} 17^{\mathrm{m}} 37^{\mathrm{s}} .000$ & $-05^{\mathrm{d}} 09^{\mathrm{m}} 12^{\mathrm{s}} \cdot 00$ & 2014 Jan 27 & 2012.1.00934.S & 222 & 1.35 & $1.64 \times 0.7$ & 58.27 \\
\hline 2 & $\begin{array}{l}\text { HiZELS-UDS- } \\
\text { NBK-1196 }\end{array}$ & $02^{\mathrm{h}} 17^{\mathrm{m}} 40^{\mathrm{s}} \cdot 120$ & $-05^{\mathrm{d}} 12^{\mathrm{m}} 02^{\mathrm{s}} .35$ & 2014 Jan 27 & 2012.1.00934.S & 222 & 1.35 & $1.65 \times 0.7$ & 60.39 \\
\hline 3 & $\begin{array}{c}\text { HiZELS-UDS- } \\
\text { NBK-1348 }\end{array}$ & $02^{\mathrm{h}} 17^{\mathrm{m}} 51^{\mathrm{s}} .470$ & $-05^{\mathrm{d}} 10^{\mathrm{m} 3} 6^{\mathrm{s}} \cdot 40$ & 2014 Jan 27 & 2012.1.00934.S & 222 & 1.35 & $1.65 \times 0.7$ & 59.21 \\
\hline 4 & $\begin{array}{c}\text { HiZELS-UDS- } \\
\text { NBK-8806 }\end{array}$ & $02^{\mathrm{h}} 17^{\mathrm{m}} 18^{\mathrm{s}} \cdot 650$ & $-05^{\mathrm{d}} 07^{\mathrm{m}} 54^{\mathrm{s}} \cdot 20$ & 2014 Jan 27 & 2012.1.00934.S & 222 & 1.35 & $1.64 \times 0.7$ & 59.53 \\
\hline 5 & Himiko & $02^{\mathrm{h}} 17^{\mathrm{m}} 57^{\mathrm{s}} .563$ & $-05^{\mathrm{d}} 08^{\mathrm{m}} 44^{\mathrm{s}} \cdot 45$ & $2012 \mathrm{Jul} 15$ & 2011.0.00115.S & 259 & 1.16 & $0.71 \times 0.48$ & 19.86 \\
\hline 6 & SXDF1100.013 & $02^{\mathrm{h}} 16^{\mathrm{m}} 46^{\mathrm{s}} \cdot 210$ & $-05^{\mathrm{d}} 03^{\mathrm{m}} 47^{\mathrm{s}} \cdot 83$ & 2013 Nov 19 & 2012.1.00326.S & 265 & 1.13 & $0.57 \times 0.38$ & 81.4 \\
\hline 7 & SXDF1100.019 & $02^{\mathrm{h}} 17^{\mathrm{m}} 15^{\mathrm{s}} \cdot 920$ & $-05^{\mathrm{d}} 04^{\mathrm{m}} 03^{\mathrm{s}} .13$ & 2013 Nov 19 & 2012.1.00326.S & 265 & 1.13 & $0.57 \times 0.38$ & 81.23 \\
\hline 8 & SXDF1100.027 & $02^{\mathrm{h}} 17^{\mathrm{m}} 20^{\mathrm{s}} \cdot 550$ & $-05^{\mathrm{d}} 08^{\mathrm{m}} 42^{\mathrm{s}} \cdot 36$ & 2013 Nov 19 & 2012.1.00326.S & 265 & 1.13 & $0.57 \times 0.38$ & 80.98 \\
\hline 9 & SXDF1100.036 & $02^{\mathrm{h}} 18^{\mathrm{m}} 00.700$ & $-05^{\mathrm{d}} 07^{\mathrm{m}} 29^{\mathrm{s}} \cdot 69$ & 2013 Nov 19 & 2012.1.00326.S & 265 & 1.13 & $0.57 \times 0.38$ & 81.5 \\
\hline 10 & SXDF1100.039 & $02^{\mathrm{h}} 18^{\mathrm{m}} 30^{\mathrm{s}} .590$ & $-05^{\mathrm{d}} 01^{\mathrm{m}} 16^{\mathrm{s}} .09$ & 2013 Nov 19 & 2012.1.00326.S & 265 & 1.13 & $0.57 \times 0.38$ & 79.86 \\
\hline 11 & SXDF1100.045 & $02^{\mathrm{h}} 18^{\mathrm{m}} 15^{\mathrm{s}} \cdot 350$ & $-04^{\mathrm{d}} 54^{\mathrm{m}} 03^{\mathrm{s}} .36$ & 2013 Nov 19 & 2012.1.00326.S & 265 & 1.13 & $0.57 \times 0.38$ & 79.46 \\
\hline 12 & SXDF1100.049 & $02^{\mathrm{h}} 17^{\mathrm{m}} 33^{\mathrm{s}} \cdot 310$ & $-04^{\mathrm{d}} 57^{\mathrm{m}} 02^{\mathrm{s}} .02$ & 2013 Nov 19 & 2012.1.00326.S & 265 & 1.13 & $0.57 \times 0.38$ & 81.66 \\
\hline 13 & SXDF1100.053 & $02^{\mathrm{h}} 16^{\mathrm{m}} 48^{\mathrm{s}} \cdot 170$ & $-04^{\mathrm{d}} 58^{\mathrm{m}} 56^{\mathrm{s}} \cdot 66$ & 2013 Nov 19 & 2012.1.00326.S & 265 & 1.13 & $0.57 \times 0.38$ & 82.26 \\
\hline 14 & SXDF1100.060 & $02^{\mathrm{h}} 17^{\mathrm{m}} 37^{\mathrm{s}} .700$ & $-05^{\mathrm{d}} 08^{\mathrm{m}} 23^{\mathrm{s}} .17$ & 2013 Nov 30 & 2012.1.00326.S & 265 & 1.13 & $0.68 \times 0.48$ & 137.39 \\
\hline 15 & SXDF1100.063 & $02^{\mathrm{h}} 17^{\mathrm{m}} 35^{\mathrm{s}} .990$ & $-04^{\mathrm{d}} 52^{\mathrm{m}} 18^{\mathrm{s}} .15$ & 2013 Nov 19 & 2012.1.00326.S & 265 & 1.13 & $0.57 \times 0.38$ & 80.8 \\
\hline 16 & SXDF1100.073 & $02^{\mathrm{h}} 18^{\mathrm{m}} 10^{\mathrm{s}} .270$ & $-05^{\mathrm{d}} 11^{\mathrm{m}} 26^{\mathrm{s}} .18$ & 2013 Nov 30 & 2012.1.00326.S & 265 & 1.13 & $0.68 \times 0.48$ & 141.14 \\
\hline 17 & SXDF1100.082 & $02^{\mathrm{h}} 17^{\mathrm{m}} 58^{\mathrm{s}} \cdot 290$ & $-04^{\mathrm{d}} 59^{\mathrm{m}} 11^{\mathrm{s}} .16$ & 2013 Nov 30 & 2012.1.00326.S & 265 & 1.13 & $0.69 \times 0.48$ & 141.18 \\
\hline 18 & SXDF1100.083 & $02^{\mathrm{h}} 17^{\mathrm{m}} 11^{\mathrm{s}} .830$ & $-05^{\mathrm{d}} 03^{\mathrm{m}} 59^{\mathrm{s}} .71$ & 2013 Nov 30 & 2012.1.00326.S & 265 & 1.13 & $0.69 \times 0.48$ & 143.26 \\
\hline 19 & SXDF1100.090 & $02^{\mathrm{h}} 17^{\mathrm{m}} 23^{\mathrm{s}} \cdot 660$ & $-04^{\mathrm{d}} 57^{\mathrm{m}} 24^{\mathrm{s}} .06$ & 2013 Nov 30 & 2012.1.00326.S & 265 & 1.13 & $0.69 \times 0.48$ & 139.67 \\
\hline 20 & SXDF1100.101 & $02^{\mathrm{h}} 18^{\mathrm{m}} 35^{\mathrm{s}} \cdot 580$ & $-05^{\mathrm{d}} 10^{\mathrm{m}} 02^{\mathrm{s}} \cdot 67$ & 2013 Nov 30 & 2012.1.00326.S & 265 & 1.13 & $0.69 \times 0.48$ & 139.46 \\
\hline 21 & SXDF1100.104 & $02^{\mathrm{h}} 18^{\mathrm{m}} 04^{\mathrm{s}} .930$ & $-05^{\mathrm{d}} 08^{\mathrm{m}} 19^{\mathrm{s}} .32$ & 2013 Nov 30 & 2012.1.00326.S & 265 & 1.13 & $0.68 \times 0.48$ & 141.65 \\
\hline 22 & SXDF1100.109 & $02^{\mathrm{h}} 18^{\mathrm{m}} 23^{\mathrm{s}} \cdot 720$ & $-05^{\mathrm{d}} 08^{\mathrm{m}} 05^{\mathrm{s}} .49$ & 2013 Nov 30 & 2012.1.00326.S & 265 & 1.13 & $0.69 \times 0.48$ & 140.09 \\
\hline 23 & SXDF1100.110 & $02^{\mathrm{h}} 17^{\mathrm{m}} 44^{\mathrm{s}} \cdot 200$ & $-05^{\mathrm{d}} 04^{\mathrm{m}} 08^{\mathrm{s}} .78$ & 2013 Nov 30 & 2012.1.00326.S & 265 & 1.13 & $0.69 \times 0.48$ & 141.69 \\
\hline 24 & SXDF1100.123 & $02^{\mathrm{h}} 18^{\mathrm{m}} 11^{\mathrm{s}} .930$ & $-05^{\mathrm{d}} 14^{\mathrm{m}} 20^{\mathrm{s}} \cdot 94$ & 2013 Nov 30 & 2012.1.00326.S & 265 & 1.13 & $0.69 \times 0.48$ & 138.85 \\
\hline 25 & SXDF1100.127 & $02^{\mathrm{h}} 17^{\mathrm{m}} 33^{\mathrm{s}} \cdot 080$ & $-04^{\mathrm{d}} 48^{\mathrm{m}} 51^{\mathrm{s}} .89$ & 2013 Nov 30 & 2012.1.00326.S & 265 & 1.13 & $0.64 \times 0.46$ & 65.92 \\
\hline 26 & SXDF1100.154 & $02^{\mathrm{h}} 18^{\mathrm{m}} 14^{\mathrm{s}} .490$ & $-04^{\mathrm{d}} 56^{\mathrm{m}} 12.80$ & 2013 Nov 30 & 2012.1.00326.S & 265 & 1.13 & $0.64 \times 0.46$ & 63.9 \\
\hline 27 & SXDF1100.174 & $02^{\mathrm{h}} 18^{\mathrm{m}} 16^{\mathrm{s}} \cdot 350$ & $-05^{\mathrm{d}} 15^{\mathrm{m}} 28^{\mathrm{s}} .80$ & 2013 Nov 30 & 2012.1.00326.S & 265 & 1.13 & $0.64 \times 0.46$ & 64.95 \\
\hline 28 & SXDF1100.179 & $02^{\mathrm{h}} 18^{\mathrm{m}} 43^{\mathrm{s}} \cdot 870$ & $-04^{\mathrm{d}} 57^{\mathrm{m}} 33^{\mathrm{s}} \cdot 62$ & 2013 Nov 30 & 2012.1.00326.S & 265 & 1.13 & $0.64 \times 0.46$ & 64.43 \\
\hline 29 & SXDF1100.230 & $02^{\mathrm{h}} 17^{\mathrm{m}} 58^{\mathrm{s}} \cdot 610$ & $-04^{\mathrm{d}} 45^{\mathrm{m}} 49^{\mathrm{s}} \cdot 13$ & 2013 Nov 30 & 2012.1.00326.S & 265 & 1.13 & $0.64 \times 0.46$ & 64.6 \\
\hline 30 & SXDF1100.231 & $02^{\mathrm{h}} 17^{\mathrm{m}} 59^{\mathrm{s}} .910$ & $-04^{\mathrm{d}} 46^{\mathrm{m}} 50^{\mathrm{s}} .05$ & 2013 Nov 30 & 2012.1.00326.S & 265 & 1.13 & $0.64 \times 0.46$ & 65.54 \\
\hline 31 & SXDF1100.233 & $02^{\mathrm{h}} 18^{\mathrm{m}} 53^{\mathrm{s}} .030$ & $-04^{\mathrm{d}} 58^{\mathrm{m}} 14^{\mathrm{s}} .74$ & 2013 Nov 19 & 2012.1.00326.S & 265 & 1.13 & $0.57 \times 0.38$ & 81.82 \\
\hline 32 & SXDF1100.250 & $02^{\mathrm{h}} 17^{\mathrm{m}} 52^{\mathrm{s}} .690$ & $-05^{\mathrm{d}} 20^{\mathrm{m}} 31^{\mathrm{s}} .72$ & 2013 Nov 30 & 2012.1.00326.S & 265 & 1.13 & $0.64 \times 0.46$ & 64.78 \\
\hline 33 & SXDF1100.253 & $02^{\mathrm{h}} 18^{\mathrm{m}} 26^{\mathrm{s}} .970$ & $-05^{\mathrm{d}} 14^{\mathrm{m}} 38^{\mathrm{s}} \cdot 61$ & 2013 Nov 30 & 2012.1.00326.S & 265 & 1.13 & $0.64 \times 0.46$ & 64.45 \\
\hline 34 & SXDF1100.276 & $02^{\mathrm{h}} 18^{\mathrm{m}} 33^{\mathrm{s}} .930$ & $-04^{\mathrm{d}} 54^{\mathrm{m}} 23^{\mathrm{s}} .85$ & 2013 Nov 30 & 2012.1.00326.S & 265 & 1.13 & $0.64 \times 0.46$ & 66.35 \\
\hline 35 & SXDF1100.277 & $02^{\mathrm{h}} 17^{\mathrm{m}} 24^{\mathrm{s}} .090$ & $-05^{\mathrm{d}} 20^{\mathrm{m}} 21^{\mathrm{s}} .94$ & 2013 Nov 30 & 2012.1.00326.S & 265 & 1.13 & $0.64 \times 0.46$ & 63.16 \\
\hline 36 & SXDF.220GHZ & $02^{\mathrm{h}} 18^{\mathrm{m}} 56^{\mathrm{s}} .536$ & $-05^{\mathrm{d}} 19^{\mathrm{m}} 58^{\mathrm{s}} .92$ & 2014 May 3 & 2012.1.00374.S & 224 & 1.33 & $0.82 \times 0.62$ & 12.56 \\
\hline 37 & SXDF-NB1 & $02^{\mathrm{h}} 18^{\mathrm{m}} 56^{\mathrm{s}} .536$ & $-05^{\mathrm{d}} 19^{\mathrm{m}} 58^{\mathrm{s}} .92$ & 2015 Dec 27 & 2013.A.00021.S & 224 & 1.33 & $1.94 \times 1.07$ & 15.1 \\
\hline 38 & SXDS1_13015 & $02^{\mathrm{h}} 17^{\mathrm{m}} 13^{\mathrm{s}} \cdot 617$ & $-05^{\mathrm{d}} 09^{\mathrm{m}} 39^{\mathrm{s}} .82$ & 2012 Aug 15 & 2011.0.00648.S & 243 & 1.23 & $0.8 \times 0.58$ & 100.87 \\
\hline 39 & SXDS1_1723 & $02^{\mathrm{h}} 17^{\mathrm{m}} 32^{\mathrm{s}} \cdot 701$ & $-05^{\mathrm{d}} 13^{\mathrm{m}} 16^{\mathrm{s}} .47$ & 2012 Aug 26 & 2011.0.00648.S & 240 & 1.25 & $1.11 \times 0.59$ & 111.28 \\
\hline 40 & SXDS1_33244 & $02^{\mathrm{h}} 16^{\mathrm{m}} 47^{\mathrm{s}} .397$ & $-05^{\mathrm{d}} 03^{\mathrm{m}} 28^{\mathrm{s}} .09$ & 2012 Aug 26 & 2011.0.00648.S & 240 & 1.25 & $0.95 \times 0.59$ & 77.72 \\
\hline 41 & SXDS1_35572 & $02^{\mathrm{h}} 17^{\mathrm{m}} 34^{\mathrm{s}} \cdot 652$ & $-05^{\mathrm{d}} 02^{\mathrm{m}} 38^{\mathrm{s}} .97$ & 2012 Aug 11 & 2011.0.00648.S & 236 & 1.27 & $0.78 \times 0.58$ & 46.17 \\
\hline 42 & SXDS1_59863 & $02^{\mathrm{h}} 17^{\mathrm{m}} 45^{\mathrm{s}} .877$ & $-04^{\mathrm{d}} 54^{\mathrm{m}} 37^{\mathrm{s}} \cdot 60$ & 2012 Aug 15 & 2011.0.00648.S & 243 & 1.23 & $0.81 \times 0.58$ & 95.02 \\
\hline 43 & SXDS1_59914 & $02^{\mathrm{h}} 17^{\mathrm{m}} 12^{\mathrm{s}} \cdot 977$ & $-04^{\mathrm{d}} 54^{\mathrm{m}} 40^{\mathrm{s}} \cdot 40$ & 2012 Aug 26 & 2011.0.00648.S & 240 & 1.25 & $1.04 \times 0.59$ & 109.0 \\
\hline 44 & SXDS1_67002 & $02^{\mathrm{h}} 19^{\mathrm{m}} 02^{\mathrm{s}} .654$ & $-04^{\mathrm{d}} 49^{\mathrm{m}} 55^{\mathrm{s}} .83$ & 2012 Aug 15 & 2011.0.00648.S & 243 & 1.23 & $0.83 \times 0.57$ & 97.8 \\
\hline 45 & SXDS1_68849 & $02^{\mathrm{h}} 17^{\mathrm{m}} 00^{\mathrm{s}} .276$ & $-04^{\mathrm{d}} 48^{\mathrm{m}} 14^{\mathrm{s}} .53$ & 2012 Aug 26 & 2011.0.00648.S & 240 & 1.25 & $0.85 \times 0.6$ & 96.21 \\
\hline 46 & SXDS1_79518 & $02^{\mathrm{h}} 18^{\mathrm{m}} 59^{\mathrm{s}} .059$ & $-04^{\mathrm{d}} 51^{\mathrm{m}} 24^{\mathrm{s}} .91$ & 2012 Aug 26 & 2011.0.00648.S & 240 & 1.25 & $0.9 \times 0.6$ & 99.08 \\
\hline 47 & SXDS1 & $02^{\mathrm{h}} 17^{\mathrm{m}} 05^{\mathrm{s}} .789$ & $-04^{\mathrm{d}} 51^{\mathrm{m}} 25^{\mathrm{s}} \cdot 68$ & 2012 Aug 9 & 2011.0.00648.S & 231 & 1.3 & $0.8 \times 0.67$ & 64.28 \\
\hline 48 & SXDS2_13316 & $02^{\mathrm{h}} 17^{\mathrm{m}} 39^{\mathrm{s}} .035$ & $-04^{\mathrm{d}} 44^{\mathrm{m}} 41^{\mathrm{s}} .75$ & 2012 Aug 15 & 2011.0.00648.S & 243 & 1.23 & $0.81 \times 0.57$ & 92.28 \\
\hline 49 & SXDS2_22198 & $02^{\mathrm{h}} 17^{\mathrm{m}} 53^{\mathrm{s}} \cdot 416$ & $-04^{\mathrm{d}} 42^{\mathrm{m}} 53^{\mathrm{s}} .42$ & 2012 Aug 11 & 2011.0.00648.S & 236 & 1.27 & $0.81 \times 0.58$ & 44.84 \\
\hline 50 & SXDS2 & $02^{\mathrm{h}} 17^{\mathrm{m}} 24^{\mathrm{s}} .356$ & $-05^{\mathrm{d}} 00^{\mathrm{m}} 44^{\mathrm{s}} .85$ & 2012 Aug 9 & 2011.0.00648.S & 231 & 1.3 & $0.8 \times 0.66$ & 63.38 \\
\hline 51 & SXDS3_101746 & $02^{\mathrm{h}} 18^{\mathrm{m}} 04^{\mathrm{s}} \cdot 178$ & $-05^{\mathrm{d}} 19^{\mathrm{m}} 38^{\mathrm{s}} .28$ & 2012 Aug 26 & 2011.0.00648.S & 240 & 1.25 & $0.93 \times 0.6$ & 99.25 \\
\hline 52 & SXDS3_103139 & $02^{\mathrm{h}} 16^{\mathrm{m}} 57^{\mathrm{s}} .652$ & $-05^{\mathrm{d}} 14^{\mathrm{m}} 34^{\mathrm{s}} .86$ & 2012 Aug 11 & 2011.0.00648.S & 236 & 1.27 & $0.77 \times 0.58$ & 46.08 \\
\hline 53 & SXDS3_110465 & $02^{\mathrm{h}} 18^{\mathrm{m}} 20^{\mathrm{s}} \cdot 953$ & $-05^{\mathrm{d}} 19^{\mathrm{m}} 07^{\mathrm{s}} .71$ & 2012 Aug 26 & 2011.0.00648.S & 240 & 1.25 & $0.99 \times 0.6$ & 99.74 \\
\hline 54 & SXDS3 & $02^{\mathrm{h}} 17^{\mathrm{m}} 13^{\mathrm{s}} \cdot 683$ & $-05^{\mathrm{d}} 04^{\mathrm{m}} 07^{\mathrm{s}} .66$ & 2012 Aug 9 & 2011.0.00648.S & 231 & 1.3 & $0.81 \times 0.66$ & 60.34 \\
\hline 55 & SXDS5_19723 & $02^{\mathrm{h}} 16^{\mathrm{m}} 24^{\mathrm{s}} .372$ & $-05^{\mathrm{d}} 09^{\mathrm{m}} 18^{\mathrm{s}} .05$ & 2012 Aug 11 & 2011.0.00648.S & 236 & 1.27 & $0.77 \times 0.58$ & 45.41 \\
\hline 56 & SXDS5_28019 & $02^{\mathrm{h}} 16^{\mathrm{m}} 08^{\mathrm{s}} .532$ & $-05^{\mathrm{d}} 06^{\mathrm{m}} 15^{\mathrm{s}} \cdot 61$ & 2012 Aug 11 & 2011.0.00648.S & 236 & 1.27 & $0.78 \times 0.58$ & 46.36 \\
\hline
\end{tabular}


Table 4

(Continued)

\begin{tabular}{|c|c|c|c|c|c|c|c|c|c|}
\hline Index & Map & $\begin{array}{c}\text { R.A. } \\
\text { (J2000) }\end{array}$ & $\begin{array}{c}\text { Decl. } \\
(\mathrm{J} 2000)\end{array}$ & $\begin{array}{l}\text { Date of } \\
\text { Observation } \\
\quad \text { (yyyy } \\
-\mathrm{mm}-\mathrm{dd})\end{array}$ & Project ID & $\begin{array}{c}\nu_{\mathrm{obs}} \\
(\mathrm{GHz})\end{array}$ & $\begin{array}{l}\lambda_{\text {obs }} \\
(\mathrm{mm})\end{array}$ & $\begin{array}{l}\text { Resolving } \\
\text { Beam } \\
\left(" \times{ }^{\prime \prime}\right)\end{array}$ & $\begin{array}{c}\text { rms Noise } \\
\left(\mu \mathrm{Jy}_{\text {beam }}^{-1}\right)\end{array}$ \\
\hline 57 & SXDS5_9364 & $02^{\mathrm{h}} 16^{\mathrm{m}} 33^{\mathrm{s}} \cdot 807$ & $-05^{\mathrm{d}} 13^{\mathrm{m}} 44^{\mathrm{s}} .68$ & 2012 Aug 15 & $2011.0 .00648 . S$ & 243 & 1.23 & $0.81 \times 0.57$ & 97.7 \\
\hline 58 & UDS16 & $02^{\mathrm{h}} 17^{\mathrm{m}} 25^{\mathrm{s}} .474$ & $-05^{\mathrm{d}} 11^{\mathrm{m}} 08^{\mathrm{s}} \cdot 25$ & 2016 Mar 31 & 2015.1.01105.S & 241 & 1.24 & $0.96 \times 0.8$ & 21.5 \\
\hline 59 & WMH5 & $02^{\mathrm{h}} 26^{\mathrm{m}} 27^{\mathrm{s}} .030$ & $-04^{\mathrm{d}} 52^{\mathrm{m}} 38^{\mathrm{s}} \cdot 30$ & 2014 Jun 16 & 2013.1.00815.S & 262 & 1.14 & $0.5 \times 0.48$ & 18.05 \\
\hline 60 & XMMF10 & $02^{\mathrm{h}} 16^{\mathrm{m}} 311^{\mathrm{s}} .850$ & $-05^{\mathrm{d}} 53^{\mathrm{m}} 22^{\mathrm{s}} \cdot 20$ & 2012 Jun 17 & 2011.0.00539.S & 343 & 0.87 & $0.51 \times 0.41$ & 220.03 \\
\hline 61 & XMMF11 & $02^{\mathrm{h}} 21^{\mathrm{m}} 35^{\mathrm{s}} \cdot 220$ & $-06^{\mathrm{d}} 26^{\mathrm{m}} 16^{\mathrm{s}} .97$ & 2012 Jun 17 & 2011.0.00539.S & 343 & 0.87 & $0.51 \times 0.41$ & 261.51 \\
\hline 62 & XMMF12 & $02^{\mathrm{h}} 22^{\mathrm{m}} 50^{\mathrm{s}} \cdot 830$ & $-03^{\mathrm{d}} 24^{\mathrm{m}} 13^{\mathrm{s}} \cdot 81$ & 2012 Jun 17 & 2011.0.00539.S & 342 & 0.87 & $0.49 \times 0.41$ & 212.05 \\
\hline 63 & XMMF13 & $02^{\mathrm{h}} 18^{\mathrm{m}} 41^{\mathrm{s}} .550$ & $-03^{\mathrm{d}} 50^{\mathrm{m}} 01^{\mathrm{s}} .90$ & 2012 Jun 17 & 2011.0.00539.S & 343 & 0.87 & $0.51 \times 0.41$ & 227.81 \\
\hline 64 & XMMF14 & $02^{\mathrm{h}} 20^{\mathrm{m}} 29^{\mathrm{s}} \cdot 140$ & $-06^{\mathrm{d}} 48^{\mathrm{m}} 45^{\mathrm{s}} .89$ & 2012 Jun 17 & 2011.0.00539.S & 343 & 0.87 & $0.51 \times 0.41$ & 236.57 \\
\hline 65 & XMMF15 & $02^{\mathrm{h}} 22^{\mathrm{m}} 05^{\mathrm{s}} \cdot 540$ & $-07^{\mathrm{d}} 07^{\mathrm{m}} 27^{\mathrm{s}} .20$ & 2012 Jun 17 & 2011.0.00539.S & 343 & 0.87 & $0.51 \times 0.41$ & 212.96 \\
\hline 66 & XMMF16 & $02^{\mathrm{h}} 19^{\mathrm{m}} 42^{\mathrm{s}} \cdot 910$ & $-05^{\mathrm{d}} 24^{\mathrm{m}} 32^{\mathrm{s}} \cdot 54$ & 2012 Jun 17 & 2011.0.00539.S & 343 & 0.87 & $0.51 \times 0.41$ & 224.83 \\
\hline 67 & XMMF17 & $02^{\mathrm{h}} 19^{\mathrm{m}} 18^{\mathrm{s}} \cdot 400$ & $-03^{\mathrm{d}} 10^{\mathrm{m}} 51^{\mathrm{s}} \cdot 30$ & 2012 Jun 17 & 2011.0.00539.S & 343 & 0.87 & $0.51 \times 0.42$ & 259.53 \\
\hline 68 & XMMF18 & $02^{\mathrm{h}} 29^{\mathrm{m}} 44^{\mathrm{s}} \cdot 790$ & $-03^{\mathrm{d}} 41^{\mathrm{m}} 10^{\mathrm{s}} .17$ & 2012 Jun 17 & 2011.0.00539.S & 343 & 0.87 & $0.52 \times 0.41$ & 300.12 \\
\hline 69 & XMMF19 & $02^{\mathrm{h}} 20^{\mathrm{m}} 21^{\mathrm{s}} \cdot 610$ & $-01^{\mathrm{d}} 53^{\mathrm{m}} 29^{\mathrm{s}} .12$ & 2012 Jun 17 & 2011.0.00539.S & 343 & 0.87 & $0.52 \times 0.42$ & 277.51 \\
\hline 70 & XMMF4 & $02^{\mathrm{h}} 20^{\mathrm{m}} 16^{\mathrm{s}} .689$ & $-06^{\mathrm{d}} 01^{\mathrm{m}} 44^{\mathrm{s}} .38$ & 2012 Jun 17 & 2011.0.00539.S & 343 & 0.87 & $0.51 \times 0.41$ & 262.64 \\
\hline 71 & XMMF5 & $02^{\mathrm{h}} 22^{\mathrm{m}} 01^{\mathrm{s}} .576$ & $-03^{\mathrm{d}} 33^{\mathrm{m}} 40^{\mathrm{s}} \cdot 60$ & 2012 Jun 17 & 2011.0.00539.S & 343 & 0.87 & $0.52 \times 0.41$ & 233.23 \\
\hline 72 & XMMF6 & $02^{\mathrm{h}} 25^{\mathrm{m}} 48^{\mathrm{s}} \cdot 210$ & $-04^{\mathrm{d}} 17^{\mathrm{m}} 51^{\mathrm{s}} \cdot 10$ & 2012 Jun 17 & 2011.0.00539.S & 343 & 0.87 & $0.51 \times 0.41$ & 254.89 \\
\hline 73 & XMMF7 & $02^{\mathrm{h}} 18^{\mathrm{m}} 53^{\mathrm{s}} .100$ & $-06^{\mathrm{d}} 33^{\mathrm{m}} 23^{\mathrm{s}} \cdot 70$ & 2012 Jun 17 & 2011.0.00539.S & 343 & 0.87 & $0.51 \times 0.41$ & 219.54 \\
\hline 74 & XMMF8 & $02^{\mathrm{h}} 30^{\mathrm{m}} 05^{\mathrm{s}} .980$ & $-03^{\mathrm{d}} 41^{\mathrm{m}} 52^{\mathrm{s}} .70$ & 2012 Jun 17 & 2011.0.00539.S & 343 & 0.87 & $0.52 \times 0.41$ & 254.45 \\
\hline
\end{tabular}

\section{ORCID iDs}

Pallavi Patil (ib https://orcid.org/0000-0002-9471-8499 Mark Lacy (1) https://orcid.org/0000-0002-3032-1783 Duncan Farrah (D) https://orcid.org/0000-0003-1748-2010 José Afonso (10 https://orcid.org/0000-0002-9149-2973 Jason Surace (ib) https://orcid.org/0000-0001-7291-0087

\section{References}

Aihara, H., Armstrong, R., Bickerton, S., et al. 2018, PASJ, 70, S8 Alaghband-Zadeh, S., Chapman, S. C., Swinbank, A. M., et al. 2012, MNRAS, 424, 2232

Alberts, S., Wilson, G. W., Lu, Y., et al. 2013, MNRAS, 431, 194

Aravena, M., Decarli, R., Walter, F., et al. 2016, ApJ, 833, 68

Aretxaga, I., Wilson, G. W., Aguilar, E., et al. 2011, MNRAS, 415, 3831

Astropy Collaboration, Robitaille, T. P., Tollerud, E. J., et al. 2013, A\&A, 558, A33

Austermann, J. E., Dunlop, J. S., Perera, T. A., et al. 2010, MNRAS, 401, 160 Banerji, M., Chapman, S. C., Smail, I., et al. 2011, MNRAS, 418, 1071 Barger, A. J., Cowie, L. L., Sanders, D. B., et al. 1998, Natur, 394, 248 Barger, A. J., Wang, W.-H., Cowie, L. L., et al. 2012, ApJ, 761, 89 Bertoldi, F., Carilli, C., Aravena, M., et al. 2007, ApJS, 172, 132

Béthermin, M., De Breuck, C., Sargent, M., \& Daddi, E. 2015, A\&A, 576, L9 Biggs, A. D., Ivison, R. J., Ibar, E., et al. 2011, MNRAS, 413, 2314 Blain, A. W., Jameson, A., Smail, I., et al. 1999, MNRAS, 309, 715 Blain, A. W., Smail, I., Ivison, R. J., Kneib, J.-P., \& Frayer, D. T. 2002, PhR, 369,111

Brammer, G. B., van Dokkum, P. G., \& Coppi, P. 2008, ApJ, 686, 1503 Brammer, G. B., Whitaker, K. E., van Dokkum, P. G., et al. 2011, ApJ, 739, 24 Brisbin, D., Miettinen, O., Aravena, M., et al. 2017, A\&A, 608, A15 Bussmann, R. S., Riechers, D., Fialkov, A., et al. 2015, ApJ, 812, 43 Carniani, S., Maiolino, R., De Zotti, G., et al. 2015, A\&A, 584, A78 Casey, C. M., Chen, C.-C., Cowie, L. L., et al. 2013, MNRAS, 436, 1919 Casey, C. M., Narayanan, D., \& Cooray, A. 2014, PhR, 541, 45

Chapin, E. L., Chapman, S. C., Coppin, K. E., et al. 2011, MNRAS, 411, 505 Chapman, S. C., Blain, A. W., Smail, I., \& Ivison, R. J. 2005, ApJ, 622, 772

Chapman, S. C., Scott, D., Borys, C., \& Fahlman, G. G. 2002, MNRAS, 330, 92

Chapman, S. C., Smail, I., Blain, A. W., \& Ivison, R. J. 2004, ApJ, 614, 671

Chen, C.-C., Cowie, L. L., Barger, A. J., et al. 2013a, ApJ, 776, 131

Chen, C.-C., Cowie, L. L., Barger, A. J., et al. 2013b, ApJ, 762, 81
Chen, C.-C., Cowie, L. L., Barger, A. J., Wang, W.-H., \& Williams, J. P. 2014, ApJ, 789, 12

Chen, C.-C., Smail, I., Ivison, R. J., et al. 2016b, ApJ, 820, 82

Chen, C.-C., Smail, I., Swinbank, A. M., et al. 2015, ApJ, 799, 194

Chen, C.-C., Smail, I., Swinbank, A. M., et al. 2016a, ApJ, 831, 91

Coppin, K., Chapin, E. L., Mortier, A. M. J., et al. 2006, MNRAS, 372, 1621

Cowie, L. L., Barger, A. J., \& Kneib, J.-P. 2002, AJ, 123, 2197

Cowie, L. L., González-López, J., Barger, A. J., et al. 2018, ApJ, 865, 106

Daddi, E., Cimatti, A., Renzini, A., et al. 2004, ApJ, 617, 746

Dole, H., Lagache, G., Puget, J.-L., et al. 2006, A\&A, 451, 417

Dunlop, J. S., McLure, R. J., Biggs, A. D., et al. 2017, MNRAS, 466, 861

Dunlop, J. S., McLure, R. J., Yamada, T., et al. 2004, MNRAS, 350, 769

Engel, H., Tacconi, L. J., Davies, R. I., et al. 2010, ApJ, 724, 233

Ezawa, H., Kawabe, R., Kohno, K., \& Yamamoto, S. 2004, Proc. SPIE, 5489, 763

Franceschini, A., Toffolatti, L., Mazzei, P., Danese, L., \& de Zotti, G. 1991 A\&AS, 89, 285

Franco, M., Elbaz, D., Béthermin, M., et al. 2018, A\&A, 620, A152

Fu, H., Cooray, A., Feruglio, C., et al. 2013, Natur, 498, 338

Fujimoto, S., Ouchi, M., Ono, Y., et al. 2016, ApJS, 222, 1

Furusawa, H., Kosugi, G., Akiyama, M., et al. 2008, ApJS, 176, 1

Geach, J. E., Chapin, E. L., Coppin, K. E. K., et al. 2013, MNRAS, 432, 53

Geach, J. E., Dunlop, J. S., Halpern, M., et al. 2017, MNRAS, 465, 1789

González-López, J., Bauer, F. E., Romero-Cañizales, C., et al. 2017, A\&A, 597, A41

Greve, T. R., Ivison, R. J., Bertoldi, F., et al. 2004, MNRAS, 354, 779

Gwyn, S. D. J. 2012, AJ, 143, 38

Hatsukade, B., Kohno, K., Aretxaga, I., et al. 2011, MNRAS, 411, 102

Hatsukade, B., Ohta, K., Seko, A., Yabe, K., \& Akiyama, M. 2013, ApJL, 769, L27

Hatsukade, B., Ohta, K., Yabe, K., et al. 2015, ApJ, 810, 91

Hauser, M. G., Arendt, R. G., Kelsall, T., et al. 1998, ApJ, 508, 25

Hopkins, A. M., Whiting, M. T., Seymour, N., et al. 2015, PASA, 32, e037

Hsu, L.-Y., Cowie, L. L., Chen, C.-C., Barger, A. J., \& Wang, W.-H. 2016 , ApJ, 829, 25

Hughes, D. H., Serjeant, S., Dunlop, J., et al. 1998, Natur, 394, 241

Ikarashi, S., Ivison, R. J., Caputi, K. I., et al. 2015, ApJ, 810, 133

Inoue, A. K., Tamura, Y., Matsuo, H., et al. 2016, Sci, 352, 1559

Ivison, R. J., Greve, T. R., Dunlop, J. S., et al. 2007, MNRAS, 380, 199

Ivison, R. J., Greve, T. R., Smail, I., et al. 2002, MNRAS, 337, 1

Ivison, R. J., Smail, I., Amblard, A., et al. 2012, MNRAS, 425, 1320

Ivison, R. J., Smail, I., Le Borgne, J.-F., et al. 1998, MNRAS, 298, 583

Jarvis, M. J., Bonfield, D. G., Bruce, V. A., et al. 2013, MNRAS, 428, 1281

Johansson, D., Sigurdarson, H., \& Horellou, C. 2011, A\&A, 527, A117

Kim, S., Wardlow, J. L., Cooray, A., et al. 2012, ApJ, 756, 28 
Knudsen, K. K., van der Werf, P. P., \& Kneib, J.-P. 2008, MNRAS, 384, 1611

Kohno, K., Yamaguchi, Y., Tamura, Y., et al. 2016, in IAU Symp.319, Galaxies at High Redshift and Their Evolution Over Cosmic Time, ed. S. Kaviraj (Cambridge: Cambridge Univ. Press), 92

Labbé, I., Huang, J., Franx, M., et al. 2005, ApJL, 624, L81

Lagache, G., Puget, J.-L., \& Dole, H. 2005, ARA\&A, 43, 727

Lang, D., Hogg, D. W., \& Schlegel, D. J. 2016, AJ, 151, 36

Laporte, N., Bauer, F. E., Troncoso-Iribarren, P., et al. 2017, A\&A, 604, A132

Laurent, G. T., Aguirre, J. E., Glenn, J., et al. 2005, ApJ, 623, 742

Lawrence, A., Warren, S. J., Almaini, O., et al. 2007, MNRAS, 379, 1599

Lonsdale, C., Polletta, M. d. C., Surace, J., et al. 2004, ApJS, 154, 54

Mauduit, J.-C., Lacy, M., Farrah, D., et al. 2012, PASP, 124, 714

McMullin, J. P., Waters, B., Schiebel, D., Young, W., \& Golap, K. 2007, in ASP Conf. Ser.376, Astronomical Data Analysis Software and Systems XVI, ed. R. A. Shaw, F. Hill, \& D. J. Bell (San Francisco, CA: ASP), 127

Michałowski, M. J., Dunlop, J. S., Koprowski, M. P., et al. 2017, MNRAS, 469, 492

Miettinen, O., Novak, M., Smolčić, V., et al. 2015, A\&A, 584, A32

Mohan, N., \& Rafferty, D. 2015, PyBDSF: Python Blob Detection and Source Finder, Astrophysics Source Code Library, ascl:1502.007

Nyland, K., Lacy, M., Sajina, A., et al. 2017, ApJS, 230, 9

Ono, Y., Ouchi, M., Kurono, Y., \& Momose, R. 2014, ApJ, 795, 5

Oteo, I., Zwaan, M. A., Ivison, R. J., Smail, I., \& Biggs, A. D. 2016, ApJ, 822,36

Ouchi, M., Ellis, R., Ono, Y., et al. 2013, ApJ, 778, 102

Patel, S. G., Kelson, D. D., Holden, B. P., Franx, M., \& Illingworth, G. D. 2011, ApJ, 735, 53

Pentericci, L., Carniani, S., Castellano, M., et al. 2016, ApJL, 829, L11

Perera, T. A., Chapin, E. L., Austermann, J. E., et al. 2008, MNRAS, 391, 1227
Pope, A., Scott, D., Dickinson, M., et al. 2006, MNRAS, 370, 1185

Rowan-Robinson, M., Babbedge, T., Oliver, S., et al. 2008, MNRAS, 386, 697

Rowan-Robinson, M., Gonzalez-Solares, E., Vaccari, M., \& Marchetti, L. 2013, MNRAS, 428, 1958

Scott, K. S., Wilson, G. W., Aretxaga, I., et al. 2012, MNRAS, 423, 575

Scott, K. S., Yun, M. S., Wilson, G. W., et al. 2010, MNRAS, 405, 2260

Sekiguchi, K. \& SXDS Team 2004, BAAS, 36, 1478

Seko, A., Ohta, K., Yabe, K., et al. 2016a, ApJ, 819, 82

Seko, A., Ohta, K., Yabe, K., et al. 2016b, ApJ, 833, 53

Simpson, J. M., Smail, I., Swinbank, A. M., et al. 2017, ApJ, 839, 58

Simpson, J. M., Swinbank, A. M., Smail, I., et al. 2014, ApJ, 788, 125

Siringo, G., Kreysa, E., Kovács, A., et al. 2009, A\&A, 497, 945

Smail, I., Ivison, R. J., \& Blain, A. W. 1997, ApJL, 490, L5

Smail, I., Ivison, R. J., Blain, A. W., \& Kneib, J.-P. 2002, MNRAS, 331, 495

Smith, D. J. B., Dunne, L., Maddox, S. J., et al. 2011, MNRAS, 416, 857

Steidel, C. C., Giavalisco, M., Pettini, M., Dickinson, M., \& Adelberger, K. L. 1996, ApJL, 462, L17

Swinbank, A. M., Simpson, J. M., Smail, I., et al. 2014, MNRAS, 438, 1267

Tokunaga, A. T., Simons, D. A., \& Vacca, W. D. 2002, PASP, 114, 180

Viero, M. P., Moncelsi, L., Quadri, R. F., et al. 2013, ApJ, 779, 32

Wardlow, J. L., Smail, I., Coppin, K. E. K., et al. 2011, MNRAS, 415, 1479

Weiß, A., Kovács, A., Coppin, K., et al. 2009, ApJ, 707, 1201

Whitaker, K. E., Labbé, I., van Dokkum, P. G., et al. 2011, ApJ, 735, 86

Williams, R. J., Quadri, R. F., Franx, M., van Dokkum, P., \& Labbé, I. 2009. ApJ, 691, 1879

Willott, C. J., Carilli, C. L., Wagg, J., \& Wang, R. 2015, ApJ, 807, 180

Willott, C. J., McLure, R. J., Hibon, P., et al. 2013, AJ, 145, 4

Wuyts, S., Labbé, I., Franx, M., et al. 2007, ApJ, 655, 51

Yamaguchi, Y., Tamura, Y., Kohno, K., et al. 2016, PASJ, 68, 82 